\title{
A Five-Equation Model for the Simulation of Interfaces between Compressible Fluids
}

\author{
Grégoire Allaire, ${ }^{*}$ Sébastien Clerc, ${ }^{+1}$ and Samuel Kokh $\dagger$ \\ *Centre de Mathématiques Appliquées, Ecole Polytechnique, 91128 Palaiseau, France; \\ and $\dagger D E N / D M 2 S / S F M E$ CEA Saclay, 91191 Gif-sur-Yvette, France \\ E-mail: gregoire.allaire@polytechnique.fr, sebastien.clerc@cea.fr, samuel.kokh@cea.fr
}

Received June 2, 2001; revised June 3, 2002

\begin{abstract}
A diffuse-interface method is proposed for the simulation of interfaces between compressible fluids with general equations of state, including tabulated laws. The interface is allowed to diffuse on a small number of computational cells and a mixture model is given for this transition region. We write conservation equations for the mass of each fluid and for the total momentum and energy of the mixture and an advection equation for the volume fraction of one of the two fluids. The model needs an additional closure law. We study two different closure laws: isobaric and isothermal. We study the mathematical properties of the resulting models: consistency, hyperbolicity, and existence of a mathematical entropy. We also study the stability of the interfaces with respect to averaging due to the numerical diffusion, a crucial property for the simulation of interface problems by conservative schemes. We show that the isobaric closure is preferable to the isothermal closure with respect to this property. We propose a Roe-type numerical scheme for the simulation of the model and show numerical results for classical test cases. (c) 2002 Elsevier Science (USA)

Key Words: multiphase flows; interface problems; real fluids.
\end{abstract}

\section{INTRODUCTION}

We consider the simulation of interfaces between two immiscible compressible fluids. Our motivation comes from the computation of water and steam two-phase flows and is discussed at length in [12-14]. This problem is modeled by the compressible Euler equations in each fluid domain and the usual jump conditions across the interface. The major numerical difficulty is to compute accurately the motion of the interface. In the literature there are mainly four possible methods for this problem. The first one is called interface tracking. It is a Lagrangian method where the interface is discretized with markers which are moved

${ }^{1}$ Current address: Alcatel Space Industries; Research Department, 100 Boulevard du Midi, 06156 Cannes La Bocca, France. 
at each time step. Examples of this approach are in $[8,33]$. The second method is called volume of fluid (VOF) and is based on an interface reconstruction process. A color function, initialized with 0 and 1 in the domains occupied by fluid 1 and 2, respectively, is advected on a fixed Eulerian grid. The actual position of the interface is recovered by a sophisticated procedure (see, e.g., $[10,15,27]$ ), which also controls the numerical diffusion. The third method is the level set method introduced by Osher and Sethian [20]. Here the interface is given as the zero level set of a continuous function, which is typically the signed distance to the interface. The interface recovery reduces to a simple interpolation problem. Methods such as front-tracking or "second-order" volume of fluid invoke a real reconstruction of the interface owing to fine interpolation processes. Despite their high accuracy, they suffer the drawback of a high complexity in three dimensions or in case of drastic topological changes of the front. On the other hand, level set methods are simple but less accurate, and sharpening techniques are used to enhance their precision (see, e.g., [21, 28]). Finally, the fourth method, to which the present work pertains, is based on a spread interface model. In this method, one does not compute the precise location of the interface. As for VOF methods each phase is marked by a color function which, because of numerical diffusion, can take intermediate values between 0 and 1 . In the transition region, these color functions are interpreted as concentrations or mass fractions, and therefore we need to introduce a mixture model or an artificial equation of state for the mixture in the transition layer which corresponds to the spread interface. This type of model was first used to compute multicomponent gas flows [2, 17] and then extended to immiscible materials (see $[1,11$, $25,26,29]$ among others). This latter approach is not contradictory with the use of level set algorithms as explained in $[13,14]$, since the color functions can be reinitialized periodically as characteristic functions valued in $\{0,1\}$ by using the usual sharpening techniques based on Hamilton-Jacobi equations (see, e.g., [21, 28]). A priori, the spread interface method is less accurate than the previous methods, but it is simpler to implement, it relies on a sound physical modeling of the mixture, and its numerical diffusion can be limited by different sharpening techniques [13]. Let us mention also some other recent numerical methods such as $[3,18]$.

In this work, we concentrate on the definition of the mixture model for the spread interface. This must be done in accordance to the following criteria:

- the model should degenerate correctly when only one fluid is present,

- the model should be hyperbolic,

- the model should conserve the total mass and energy of the fluids, and

- the model should not create spurious numerical oscillations at the interface.

In the case of two perfect gases, the common practice is to define an equivalent perfect gas for the mixture by simply averaging the coefficients of the equation of state with weights proportional to the color function (see, e.g., [2]). More precisely, the right quantity to average is $1 /(\gamma-1)$, where $\gamma$ is the adiabatic exponent of the perfect gas. Abgrall [1] and Karni [11] showed that it was desirable to include the last property of the aforementioned list, namely, the preservation of constant pressure equilibriums. Mixing two fluids with the same pressure should result in a mixture state with the same pressure. Failing to comply with this requirement leads to the generation of spurious acoustic waves near the interface; see also [5]. Based on this principle, Abgrall and Karni showed that it is necessary to give up the conservative character of the equations and proposed a quasi-conservative approach. Indeed, they introduce a nonconservative transport equation for the quantity $1 /(\gamma-1)$. Saurel and 
Abgrall [26] and Shyue [29] further extended this model to the so-called stiffened gas equation of state or to the van der Waals equation of state [30]. One of the main ideas of these extensions is to add as many transport equations as there are parameters defining the equation of state (like the adiabatic exponent $\gamma$ for a perfect gas). Therefore, the practical complexity of this approach increases with the number of parameters required to define the equation of state. In the present work, we further extend the spread interface model to a wide range of equations of state, not necessarily parametrized by few parameters (as, for example, tabulated equations of state, which are of common use in practical applications; see Section 8.6). The key idea is to replace algebraic closure relations in the mixture by a single supplementary transport equation. In particular, the number of equations of our model does not depend on the equation of state (as was the case with the previous extensions). This also has the advantage that our model conserves mass for each fluid.

The contents of the paper are the following. In Section 2 we recall the original quasiconservative model of Abgrall and Karni, which is specifically designed for a perfect gas. In one dimension (1-D) it is a system of four equations which involves a special modeling and numerical treatment of the adiabatic exponent $\gamma$. This model can be generalized to other types of gas with simple parametrized equation of state (for example, stiffened gas [26, 29] or van der Waals gas [30]). In Section 3 we extend the previous approach by considering a five-equation model which allows us to treat any type of equations of state. In Sections 4 and 5 we investigate two algebraic closures for our five-equation model: isopressure and isothermal closure respectively. For the isopressure closure, we prove in Section 6 a stability property for the pressure at the interface. In Section 7 we propose a numerical scheme for the simulation of the five-equation model. The scheme is based on Roe's approximate Riemann solver, with extensions to treat nonconservative terms and general equations of state. Numerical results are presented in Section 8 and a short conclusion is drawn in Section 9.

To conclude this introduction, let us remark that there exist more complicated models for interface simulations. In [25] Saurel presented a seven-equation model for mixtures of various fluids. The main results of this paper have been announced in [4]. We have learned recently that the authors of [19] proposed a similar model independently.

\section{THE FOUR-EQUATION MODEL}

In this section we describe the so-called four-equation model for the modeling of the mixture of two perfect gases in a thin layer around their true interface. It amounts to a simple isothermal mixing model of perfect gases, as described in [1].

Each fluid $i=1,2$ is assumed to be compressible with a density $\rho_{i}$, a specific internal energy $\varepsilon_{i}$, a pressure $P_{i}$, and a temperature $T_{i}$ satisfying the perfect gas equation of state

$$
P_{i}=\left(\gamma_{i}-1\right) \rho_{i} \varepsilon_{i}, \quad \varepsilon_{i}=c_{v i} T_{i},
$$

where $\gamma_{i}=c_{p i} / c_{v i}$ is the ratio of the specific heat capacity at constant pressure and volume, respectively, of the $i$ th fluid. We also define the specific enthalpy of fluid $i$ by $h_{i}=\varepsilon_{i}+P_{i} / \rho_{i}$. Throughout this paper, both fluids in the mixture are supposed to share the same velocity $\mathbf{u}$. We introduce a color function $z \in[0,1]$ and define the volume fractions $z_{1}=z$ and $z_{2}=1-z$. By volume averaging, we then define global quantities and thermodynamical parameters for the mixture, displayed in Table I. Note that the definition 
TABLE I

Global Variables of the Fluid Mixture

\begin{tabular}{ll}
\hline$z_{1}=z, z_{2}=1-z$ & Volume fractions \\
$\rho=z_{1} \rho_{1}+z_{2} \rho_{2}$ & Density \\
$y_{i}=z_{i} \rho_{i} / \rho$ & Mass fractions \\
$P=z_{1} P_{1}+z_{2} P_{2}$ & Pressure \\
$\rho \varepsilon=z_{1} \rho_{1} \varepsilon_{1}+z_{2} \rho_{2} \varepsilon_{2}$ & Internal energy \\
$\rho h=z_{1} \rho_{1} h_{1}+z_{2} \rho_{2} h_{2}=\rho \varepsilon+P$ & Specific enthalpy \\
$e=\varepsilon+|\mathbf{u}|^{2} / 2$ & Specific total energy \\
$H=e+P / \rho$ & Specific total enthalpy \\
\hline
\end{tabular}

of the global pressure $P$ is just Dalton's law. During the computation, there will be cells containing both fluids, and we need to define a new equation of state for this mixture if we want to treat it as a single equivalent fluid. Although this is a purely numerical artifact we use physically sound arguments to model this mixture layer. We make the assumption of a thermal equilibrium; i.e., in the area where both species are present, they share the same temperature $T=T_{1}=T_{2}$.

This isothermal assumption allows us to relate the global parameters $P, T, \rho, \varepsilon$. Indeed, introducing the mass fractions $y_{i}=z_{i} \rho_{i} / \rho$, we obtain thermodynamic parameters for the mixture

$$
\begin{aligned}
c_{p} & =y_{1} c_{p_{1}}+y_{2} c_{p_{2}}, \\
c_{v} & =y_{1} c_{v 1}+y_{2} c_{v 2}, \\
\gamma & =\frac{y_{1} c_{p_{1}}+y_{2} c_{p_{2}}}{y_{1} c_{v 1}+y_{2} c_{v 2}},
\end{aligned}
$$

such that

$$
\varepsilon=c_{v} T, \quad P=(\gamma-1) \rho \varepsilon
$$

Therefore, the mixture state equation is similar to that of a perfect gas, except that the $\gamma$ parameter depends on the mass fractions. This mixing process can be applied to other types of gas, e.g., a stiffened gas [25], but not to any real gas. There are other possible mixing laws which allow us to define a state equation for the mixture (see, e.g., the iso-heat-deposition closure law introduced by Lagoutière [16]).

Abgrall [1] and Karni [11] showed that if the average $\gamma$ is computed directly from Eq. (3), spurious pressure oscillations are created at the interface. To avoid these oscillations, Abgrall [1] introduced a parameter $\xi$ defined by

$$
\xi=\frac{1}{\gamma-1}
$$

and wrote a transport equation for $\xi$. Therefore, the four-equation model is

$$
\left\{\begin{array}{l}
\frac{\partial \rho}{\partial t}+\operatorname{div}(\rho \mathbf{u})=0, \\
\frac{\partial \rho \mathbf{u}}{\partial t}+\operatorname{div}(\rho \mathbf{u} \otimes \mathbf{u}+P \mathrm{Id})=0, \\
\frac{\partial \rho e}{\partial t}+\operatorname{div}(\rho H \mathbf{u})=0, \\
\frac{\partial \xi}{\partial t}+\mathbf{u} \cdot \operatorname{grad} \xi=0 .
\end{array}\right.
$$


Note that the last (nonconservative) equation of (5) can be put in conservative form by combining it with mass conservation. However, for numerical purposes it must be discretized as nonconservative.

Model (5) allows the recovery of the partial densities $z_{i} \rho_{i}$, partial pressures $z_{i} P_{i}$, and partial internal energies $z_{i} \varepsilon_{i}$ of each species from the knowledge of the mixture variables. Nevertheless, we cannot deduce the volume fractions $z_{i}$ or the individual thermodynamic quantities $\rho_{i}, P_{i}, \varepsilon_{i}$ from (5) (except if we add another closure relation like e.g., $P_{1}=P_{2}$ ).

The four-equation model is unfortunately restricted to very specific equation of states (EOSs) such as the perfect gas EOS. The rest of this paper is devoted to its generalization to real fluids, for which we add a supplementary transport equation. In particular, this will allow us to recover all individual thermodynamic quantities as well as the volume fractions of each phase.

\section{THE FIVE-EQUATION MODEL}

\subsection{Definition}

We keep the same notation as in the previous section (see Table I). Our model is based on the usual conservation laws for the mixture (mass, momentum, and energy balance) supplemented with one phase mass conservation equation and a transport equation for the volume fraction $z$. Therefore, the five-equation system reads

$$
\begin{aligned}
\frac{\partial z_{1} \rho_{1}}{\partial t}+\operatorname{div}\left(z_{1} \rho_{1} \mathbf{u}\right) & =0, \\
\frac{\partial z_{2} \rho_{2}}{\partial t}+\operatorname{div}\left(z_{2} \rho_{2} \mathbf{u}\right) & =0, \\
\frac{\partial \rho \mathbf{u}}{\partial t}+\operatorname{div}(\rho \mathbf{u} \otimes \mathbf{u}+P \mathrm{Id}) & =0, \\
\frac{\partial \rho e}{\partial t}+\operatorname{div}(\rho H \mathbf{u}) & =0, \\
\frac{\partial z}{\partial t}+\mathbf{u} \cdot \operatorname{grad} z & =0 .
\end{aligned}
$$

System (6)-(10) is different from the previous four-equation model (5) since one keeps track of the volume fractions, not merely the mass fractions. Its form is closer in spirit to the usual models of homogenized two-phase flows based on volume fractions (see, e.g., [24, $25,32])$.

This five-equation model is not closed. Indeed, there are five equations (in 1-D, or seven equations in 3-D) and six variables (in 1-D, or eight variables in 3-D), which are $z, \rho_{1}, \rho_{2}$, $\mathbf{u}, \varepsilon_{1}, \varepsilon_{2}$. Therefore an extra relation is needed to complete it. A possible way to understand how this missing closure relation acts in the system is to recognize that it is not possible to compute the pressure $P$ with the sole knowledge of the conserved quantities in (6) (10). Indeed, these conserved quantities yield the values of $z, \rho_{1}, \rho_{2}$, $\mathbf{u}$ but not those of $\varepsilon_{1}, \varepsilon_{2}$ (merely a linear combination of them is known). Therefore, this extra constitutive hypothesis can be thought of as a definition of a "generalized EOS" for the mixture. Of course, there are many possible closure relations that allow recovery of the pressure $P$, but among them we select those that allow recovery of all the thermodynamic variables of each 
fluid (it is easy to see that not all of them have this property). We call this selection principle a consistency property since it is physically, as well as numerically, sound to be able to characterize completely each fluid in such a mixture model.

We suggest two different closure laws based on reasonable physical assumptions that we analyze from a mathematical and numerical point of view: the isobaric closure and the isothermal closure. For each of these we study the consistency and the hyperbolicity of the system, as well as some qualitative properties of interface advection. In this study the numerical aspect of the five-equation system will be especially focused on the isobaric closure.

Remark. If the color function $z$ takes only the values 0 or 1 , which means that it describes a sharp interface, then Eqs. (6), (7), and (10) are redundant (only two out of them are independent). In such a case, the five-equation model is equivalent to the four-equation model, namely,

$$
\begin{aligned}
\frac{\partial \rho}{\partial t}+\operatorname{div}(\rho \mathbf{u}) & =0, \\
\frac{\partial \rho \mathbf{u}}{\partial t}+\operatorname{div}(\rho \mathbf{u} \otimes \mathbf{u}+P \mathrm{Id}) & =0, \\
\frac{\partial \rho e}{\partial t}+\operatorname{div}(\rho H \mathbf{u}) & =0, \\
\frac{\partial z}{\partial t}+\mathbf{u} \cdot \operatorname{grad} z & =0 .
\end{aligned}
$$

However, as soon as $z$ takes intermediate values, these two systems are not equivalent.

Remark. One advantage of the five-equation model is that it is always mass conservative with respect to each phase. In particular, whatever the numerical treatment of the color function $z$ (which is governed by a nonconservative equation), we shall never lose the important property of phase mass conservation. This is especially crucial if one couples this model with a numerical procedure of front-sharpening or antidiffusion for the color function $z$ (see [12]). Note that the total momentum and total energy are also conservative quantities, whereas each phase energy is usually not conserved.

Remark. The model can be extended to treat more than two fluids by adding a mass conservation equation and a volume fraction advection equation for each new fluid.

\subsection{Primitive Variable Formulation}

A general closure relationship for the five-equation system is the definition of a mixture equation of state of the type $P=P\left(z, \rho_{1} z, \rho_{2}(1-z), \rho \varepsilon\right)$. This formula is general enough since it depends on all thermodynamic variables in the system (but not on the velocity as is usual). Before turning to the study of specific closures of the system, we derive some evolution equations for its primitive variables (which will prove useful in the following) and show that it is hyperbolic under a mild assumption on the speed of sound.

We denote by $D \phi / D t$ the material derivative of a quantity $\phi$, i.e.,

$$
\frac{D \phi}{D t}=\frac{\partial \phi}{\partial t}+\mathbf{u} \cdot \operatorname{grad} \phi
$$


Obviously, (6) and (7) are formally equivalent to

$$
\frac{D \rho_{i} z_{i}}{D t}=-\rho_{i} z_{i} \operatorname{div}(\mathbf{u}), \quad i=1,2 .
$$

Classically, (8) can be replaced by

$$
\rho \frac{D \mathbf{u}}{D t}+\operatorname{grad} P=0
$$

while, since $\rho \varepsilon=\rho e-\rho|\mathbf{u}|^{2} / 2$, relation (9) is equivalent to

$$
\frac{D \rho \varepsilon}{D t}=-\rho h \operatorname{div}(\mathbf{u}) .
$$

Then, we write an evolution equation for the pressure. Since the total pressure is a function of the variables $z, \rho_{1} z, \rho_{2}(1-z)$, and $\rho \varepsilon$, its differential is

$$
d P=\left(\frac{\partial P}{\partial \rho \varepsilon}\right) d(\rho \varepsilon)+\sum_{i}\left(\frac{\partial P}{\partial \rho_{i} z_{i}}\right) d\left(\rho_{i} z_{i}\right)+\sum_{i}\left(\frac{\partial P}{\partial z_{i}}\right) d z_{i} .
$$

Since $D z_{i} / D t=0$, the evolution of the pressure is governed by

$$
\frac{D P}{D t}=-\left[\rho h\left(\frac{\partial P}{\partial \rho \varepsilon}\right)+\sum_{i} \rho_{i} z_{i}\left(\frac{\partial P}{\partial \rho_{i} z_{i}}\right)\right] \operatorname{div}(\mathbf{u}) .
$$

PROPOSITION 3.1. Suppose that the closure relation and the fluid equations of state are such that

$$
c^{2}=\left(\frac{\partial P}{\partial \rho \varepsilon}\right) h+\sum_{i} y_{i}\left(\frac{\partial P}{\partial \rho_{i} z_{i}}\right)>0
$$

then the five-equation model is hyperbolic.

Proof. Let us write the system of equations in the primitive variables $\left(\rho_{i} z_{i}, \mathbf{u}, P, z_{i}\right)$ :

$$
\begin{aligned}
\frac{D \rho_{i} z_{i}}{D t}+\rho_{i} z_{i} \operatorname{div}(\mathbf{u}) & =0, \\
\frac{D \mathbf{u}}{D t}+\frac{1}{\rho} \operatorname{grad} P & =0, \\
\frac{D P}{D t}+\rho c^{2} \operatorname{div}(\mathbf{u}) & =0, \\
\frac{D z_{i}}{D t} & =0 .
\end{aligned}
$$

In this form, it is obvious that the system is hyperbolic. In one space dimension for two fluids, the Jacobian matrix of the system is

$$
\left(\begin{array}{ccccc}
u & 0 & \rho_{1} z_{1} & 0 & 0 \\
0 & u & \rho_{2} z_{2} & 0 & 0 \\
0 & 0 & u & 1 / \rho & 0 \\
0 & 0 & \rho c^{2} & u & 0 \\
0 & 0 & 0 & 0 & u
\end{array}\right) .
$$


This matrix is easily shown to be diagonalizable with eigenvalues $u-c, u$ (three times), and $u+c$ and a complete set of eigenvectors, which proves the hyperbolicity (see Proposition 7.2 for a similar computation).

Finally, we give an evolution equation for the entropies that will be used in the following to investigate the existence of a mathematical entropy of the system.

LEMMA 3.1. Let $S_{i}$ be the physical entropy of phase $i$. Then, we have

$$
\sum_{i} \rho_{i} z_{i} T_{i} \frac{D S_{i}}{D t}=0
$$

Proof. We write Eq. (15) in terms of the entropy of each fluid. Since $D z_{i} / D t=0$, we first rewrite

$$
\frac{D \rho \varepsilon}{D t}=\sum_{i} \frac{D}{D t}\left(z_{i} \rho_{i} \varepsilon_{i}\right)=\sum_{i} z_{i} \frac{D \rho_{i} \varepsilon_{i}}{D t}
$$

We then use Gibb's relation to get

$$
d\left(\rho_{i} \varepsilon_{i}\right)=\rho_{i} d \varepsilon_{i}+\varepsilon_{i} d \rho_{i}=\rho_{i} T_{i} d S_{i}+h_{i} d \rho_{i} .
$$

Thus

$$
\sum_{i} \rho_{i} z_{i} T_{i} \frac{D S_{i}}{D t}+z_{i} h_{i} \frac{D \rho_{i}}{D t}=-\rho h \operatorname{div}(\mathbf{u}) .
$$

Since $D \rho_{i} / D t=-\rho_{i} \operatorname{div}(\mathbf{u})$, we obtain the desired result.

\section{ISOBARIC CLOSURE}

\subsection{Definition}

To close system (6)-(10) we supplement it with an algebraic relation of equal phase pressures. The EOSs for both fluids are supposed to be given as

$$
\rho_{i} \varepsilon_{i}:\left(\rho_{i}, P_{i}\right) \mapsto\left(\rho_{i} \varepsilon_{i}\right)\left(\rho_{i}, P_{i}\right)
$$

We define the partial derivative

$$
\xi_{i}=\left(\frac{\partial \rho_{i} \varepsilon_{i}}{\partial P_{i}}\right)_{\rho_{i}}
$$

The isobaric closure assumption reads

$$
P_{1}=P_{2}=P \text {. }
$$

We now turn to the properties of the five-equation system with isobaric closure. 


\subsection{Consistency}

In great generality we are going to make some assumptions on the EOS to prove that the five-equation model is consistent and hyperbolic. For some specific examples these assumptions are not required (see Section 4.5). We make the hypothesis that for each fluid

$$
P_{i} \geq 0 \quad \text { and } \quad P_{i}=0 \quad \text { if } \rho_{i} \varepsilon_{i}=0 \quad \text { and } \quad \xi_{i}>0 .
$$

For a perfect gas EOS, $\xi_{i}=1 /\left(\gamma_{i}-1\right)$, so the condition $\xi_{i}>0$ reads $\gamma_{i}>1$. This condition is always met by perfect gases. However, for real fluids, the condition $\xi_{i}>0$ is somewhat restrictive. A well-known counterexample is that of liquid water between 0 and $4{ }^{\circ} \mathrm{C}$.

We emphasize that assumption (24) is sufficient but not absolutely necessary. For example, $P_{i} \neq 0$ when $\rho_{i} \varepsilon_{i}=0$ for a stiffened gas, but our model is well-posed in such a case (see Section 4.5). The only merit of (24) is that it is a simple technical assumption for the following result.

PROPOSITION 4.1. Under hypothesis (24) the isobaric closure (23) allows recovery of a single value of the pressure $P$ for the five-equation model (6)-(10).

Proof. We just need to check that for given positive values of $\rho \varepsilon, \rho_{1}, \rho_{2}$ and $z \in[0,1]$ the system

$$
\begin{aligned}
P_{1}\left(\rho_{1}, \rho_{1} \varepsilon_{1}\right)-P_{2}\left(\rho_{2}, \rho_{2} \varepsilon_{2}\right) & =0, \\
z \rho_{1} \varepsilon_{1}+(1-z) \rho_{2} \varepsilon_{2} & =\rho \varepsilon
\end{aligned}
$$

admits a unique solution $\left(\rho_{1} \varepsilon_{1}, \rho_{2} \varepsilon_{2}\right)$. Defining $x=\left(\rho_{1} z_{1} \varepsilon_{1}\right) /(\rho \varepsilon)$, we introduce a function $\Phi:[0,1] \rightarrow \mathbb{R}$, defined by

$$
\Phi(x)=P_{1}\left(\rho_{1}, \frac{x}{z} \rho \varepsilon\right)-P_{2}\left(\rho_{2}, \frac{1-x}{1-z} \rho \varepsilon\right) .
$$

Assuming $P_{1}$ and $P_{2}$ to be $\mathcal{C}^{1}$, then we obtain by the chain rule

$$
\Phi^{\prime}(x)=\rho \varepsilon\left\{\frac{1}{z}\left(\frac{\partial P_{1}}{\partial \rho_{1} \varepsilon_{1}}\right)_{\rho_{1}}\left(\rho_{1}, \frac{x}{z} \rho \varepsilon\right)+\frac{1}{1-z}\left(\frac{\partial P_{2}}{\partial \rho_{2} \varepsilon_{2}}\right)_{\rho_{2}}\left(\rho_{2}, \frac{1-x}{1-z} \rho \varepsilon\right)\right\}
$$

which gives with the above notation

$$
\Phi^{\prime}(x)=\rho \varepsilon\left\{\frac{1}{z \xi_{1}}+\frac{1}{(1-z) \xi_{2}}\right\}
$$

Owing to hypothesis (24) we have $\Phi^{\prime}(x)>0, \forall x \in[0,1]$. Moreover, we have

$$
\Phi(1)=P_{1}\left(\rho_{1}, \rho \varepsilon\right) \geq 0 \text { and } \Phi(0)=-P_{2}\left(\rho_{2}, \rho \varepsilon\right) \leq 0 .
$$

The assumption on $P_{i}$ then provides the existence of $x_{0} \in[0,1]$ such that $\Phi\left(x_{0}\right)=0$. Setting $\rho_{1} \varepsilon_{1}=x_{0} \rho \varepsilon / z$ and $\rho_{2} \varepsilon_{2}=\left(1-x_{0}\right) \rho \varepsilon /(1-z)$ defines the unique solution to our problem. 
Remark. Under the isobaric closure assumption, it is straightforward to check that the datum of the global conserved quantities $z \rho_{1},(1-z) \rho_{2}, \rho \mathbf{u}, \rho e, z$ of the five-equation model (6)-(10) yields the values of all individual fluid variables $z, \rho_{1}, \rho_{2}, \mathbf{u}, \varepsilon_{1}, \varepsilon_{2}$. This is precisely what we call the consistency property of the closure law.

We now give a more precise formula than (16) for the pressure differential.

PROPOSITION 4.2. The pressure $P$ is a function of $z, z \rho_{1},(1-z) \rho_{2}, \rho \varepsilon$ and its differential is

$$
d P=\frac{1}{\xi} d(\rho \varepsilon)-\sum_{i} \frac{\delta_{i}}{\xi} d\left(z_{i} \rho_{i}\right)+M d z,
$$

with $\xi=z_{1} \xi_{1}+z_{2} \xi_{2}$ and

$$
\delta_{i}=\left(\frac{\partial \rho_{i} \varepsilon_{i}}{\partial \rho_{i}}\right)_{P_{i}}, \quad M=\frac{1}{\xi}\left(\rho_{1} \delta_{1}-\rho_{1} \varepsilon_{1}\right)-\frac{1}{\xi}\left(\rho_{2} \delta_{2}-\rho_{2} \varepsilon_{2}\right) .
$$

Proof. Recalling the definition of the mixture internal energy (see Table I), we see that its differential is given by

$$
d(\rho \varepsilon)=\sum_{i} \rho_{i} \varepsilon_{i} d z_{i}+\sum_{i} z_{i} d\left(\rho_{i} \varepsilon_{i}\right)=\sum_{i} \rho_{i} \varepsilon_{i} d z_{i}+\sum_{i} z_{i}\left[\delta_{i} d \rho_{i}+\xi_{i} d P\right]
$$

and finally

$$
d P=\frac{1}{\xi} d(\rho \varepsilon)-\sum_{i} \frac{z_{i} \delta_{i}}{\xi} d \rho_{i}-\left(\frac{\rho_{1} \varepsilon_{1}-\rho_{2} \varepsilon_{2}}{\xi}\right) d z .
$$

Using $z_{i} d \rho_{i}=d\left(z_{i} \rho_{i}\right)-\rho_{i} d z_{i}(28)$ yields formula (27).

\subsection{Hyperbolicity}

We turn to the well-posedness of the five-equation system with isobaric closure. By virtue of Proposition 3.1, it is enough to check that the mixture sound velocity $c$ is well defined, namely, that

$$
c^{2}=\left(\frac{\partial P}{\partial \rho \varepsilon}\right) h+\sum_{i} y_{i}\left(\frac{\partial P}{\partial \rho_{i} z_{i}}\right)>0 .
$$

Using the partial derivatives of $P$ given by Proposition 4.2, we easily get

$$
\xi c^{2}=h-\sum_{i} y_{i} \delta_{i}=\sum_{i} y_{i}\left(h_{i}-\delta_{i}\right)
$$

Recalling that $h_{i}=\varepsilon_{i}+P_{i} / \rho_{i}$, we find that the speed of sound $c_{i}$ of the pure fluid $i$ is given by

$$
c_{i}^{2}=\left(h_{i}-\delta_{i}\right) / \xi_{i},
$$

and thus we deduce

$$
\xi c^{2}=\sum_{i} y_{i} \xi_{i} c_{i}^{2}
$$


In other words, $c$ is some kind of average of the phase speeds of sound $c_{i}$. We can thus state the main well-posedness property of the five-equation model with isobaric closure.

PROPOSITION 4.3. Under assumption (24), and if the sound velocity $c_{i}$ of fluid $i$ is well defined, the five-equation model with isobaric closure it hyperbolic.

\subsection{Existence of a Mathematical Entropy}

In general, it is not possible to find an integrating factor in the equation of Lemma 3.1,

$$
\sum_{i} \rho_{i} z_{i} T_{i} \frac{D S_{i}}{D t}=0
$$

Therefore, we did not find a mathematical entropy for the isobaric model. However, if all the fluids in the mixture are perfect gases, we can write

$$
\rho_{i} T_{i}=P_{i} / r_{i}=P / r_{i}
$$

where $r_{i}=c_{p i}-c_{v i}$. Setting $S=\sum_{i} z_{i} S_{i} / r_{i}$, we deduce

$$
\frac{D S}{D t}=0
$$

which means that $S$ is a mathematical entropy satisfying

$$
\frac{\partial \rho S}{\partial t}+\operatorname{div}(\rho S \mathbf{u})=0
$$

\subsection{Isobaric Closure Examples}

For a general EOS, recovering the value of the pressure $P$ from the conserved quantities $\left(\rho \varepsilon, \rho_{1}, \rho_{2}, z\right)$ requires solving the nonlinear algebraic equation (25) by some type of iterative method. Such a procedure may be costly but is quite common when working with single-phase tabulated EOSs (see Section 8.6 for more details). Nevertheless, there are a number of important cases where we found an explicit expression of the pressure $P$, thus avoiding both the use of iterative root solvers for (25) and the need of the technical assumption (24).

4.5.1. Stiffened gases. We first consider the simple case of a mixture of two fluids which are so-called stiffened gases. Each fluid $i$ is characterized by its equation of state

$$
P_{i}=\left(\gamma_{i}-1\right) \rho_{i} \varepsilon_{i}-\gamma_{i} \pi_{i}
$$

involving two thermodynamic constants $\gamma_{i}>1$ (the adiabatic exponent) and $\pi_{i} \geq 0$ (a reference pressure). The coefficients we introduced here read

$$
\xi_{i}=\left(\frac{\partial \rho_{i} \varepsilon_{i}}{\partial P_{i}}\right)_{\rho_{i}}=\frac{1}{\gamma_{i}-1}, \quad \delta_{i}=\left(\frac{\partial \rho_{i} \varepsilon_{i}}{\partial \rho_{i}}\right)_{P_{i}}=0
$$


In this particular situation we obtain an analytical expression for the pressure $P=P_{1}=P_{2}$. Replacing the expression of $\rho_{i} \varepsilon_{i}$ in (30) into the definition of $\rho \varepsilon$ yields

$$
\rho \varepsilon=\sum_{i} z_{i} \rho_{i} \varepsilon_{i}=\sum_{i} z_{i}\left(\frac{P+\gamma_{i} \pi_{i}}{\gamma_{i}-1}\right)=P \sum_{i} \frac{z_{i}}{\gamma_{i}-1}+\sum_{i} z_{i} \frac{\gamma_{i} \pi_{i}}{\gamma_{i}-1} .
$$

Therefore, introducing averaged mixture coefficients, we are able to derive an explicit generalized analytic EOS for the mixture, given by

$$
\begin{aligned}
\xi & =\frac{1}{\gamma-1}=\sum_{i} \frac{z_{i}}{\gamma_{i}-1}, \\
\frac{\pi \gamma}{\gamma-1} & =\sum_{i} z_{i} \frac{\pi_{i} \gamma_{i}}{\gamma_{i}-1}, \\
P(z, \rho \varepsilon) & =(\gamma-1) \rho \varepsilon-\gamma \pi .
\end{aligned}
$$

In this particular case the five-equation system can be reduced to a classical four-equation bifluid system, equivalent to Shyue's model [29],

$$
\left\{\begin{array}{l}
\frac{\partial \rho}{\partial t}+\operatorname{div}(\rho \mathbf{u})=0 \\
\frac{\partial \rho \mathbf{u}}{\partial t}+\operatorname{div}(\rho \mathbf{u} \otimes \mathbf{u}+P \mathrm{Id})=0 \\
\frac{\partial \rho e}{\partial t}+\operatorname{div}(\rho H \mathbf{u})=0 \\
\frac{\partial z}{\partial t}+\mathbf{u} \cdot \operatorname{grad} z=0
\end{array}\right.
$$

where $P=P(z, \rho \varepsilon)$ is given by (34). In other words, knowledge of the phase densities $\rho_{i}$ is not necessary to compute the pressure. Of course, when reduced to four equations, this model no longer furnishes the phase densities or mass fractions.

4.5.2. Generalized van der Waals gases. We now consider a mixture of two generalized van der Waals gases. Both fluids are equipped with the following EOS:

$$
P_{i}\left(\rho_{i}, \rho_{i} \varepsilon_{i}\right)=\left(\frac{\gamma_{i}-1}{1-b_{i} \rho_{i}}\right)\left(\rho_{i} \varepsilon_{i}-\pi_{i}+a_{i} \rho_{i}^{2}\right)-\left(\pi_{i}+a_{i} \rho_{i}^{2}\right) .
$$

The coefficients $\delta_{i}$ and $\xi_{i}$ here read

$$
\begin{aligned}
\delta_{i} & =\left(\frac{\partial \rho_{i} \varepsilon_{i}}{\partial \rho_{i}}\right)_{P_{i}}=2 a_{i} \rho_{i}\left(\frac{1-b_{i} \rho_{i}}{\gamma_{i}-1}-1\right)-\frac{b_{i}}{\gamma_{i}-1}\left(P_{i}+\pi_{i}+a_{i} \rho_{i}^{2}\right), \\
\xi_{i} & =\left(\frac{\partial \rho_{i} \varepsilon_{i}}{\partial P_{i}}\right)_{\rho_{i}}=\frac{1-b_{i} \rho_{i}}{\gamma_{i}-1} .
\end{aligned}
$$

An explicit expression for $P$ is again available. We write $\rho \varepsilon$ using (35) as

$$
\rho \varepsilon=\sum_{i} z_{i} \rho_{i} \varepsilon_{i}=\sum_{i} z_{i}\left(\frac{1-b_{i} \rho_{i}}{\gamma_{i}-1}\right)\left(P+\pi_{i}+a_{i} \rho_{i}^{2}\right)+\sum_{i} z_{i}\left(\pi_{i}-a_{i} \rho_{i}^{2}\right) .
$$


This yields the following expression of the pressure $P$ :

$$
\begin{aligned}
P\left(\rho_{1}, \rho_{2}, \rho \varepsilon, z\right)= & {\left[\sum_{i} z_{i}\left(\frac{1-b_{i} \rho_{i}}{\gamma_{i}-1}\right)\right]^{-1}\left[\rho \varepsilon+\sum_{i} z_{i}\left(a_{i} \rho_{i}^{2}-\pi_{i}\right)\right.} \\
& \left.-\sum_{i} z_{i}\left(\frac{1-b_{i} \rho_{i}}{\gamma_{i}-1}\right)\left(\pi_{i}+a_{i} \rho_{i}^{2}\right)\right] .
\end{aligned}
$$

Unlike the previous example, the five-equation model cannot be reduced to four equations, as the full set of equations is needed to express all the variables which determine $P$. For van der Waals gases, the system is not equivalent to Shyue's extension [30].

4.5.3. Mie-Gruneisen materials. Finally, we consider a mixture of two Mie-Gruneisen fluids with the EOS

$$
P_{i}\left(\rho_{i}, \rho_{i} \varepsilon_{i}\right)=\left(\gamma_{i}\left(\rho_{i}\right)-1\right) \rho_{i} \varepsilon_{i}-\gamma_{i}\left(\rho_{i}\right) \pi_{i}\left(\rho_{i}\right)
$$

The coefficients $\xi_{i}$ and $\delta_{i}$ are now

$$
\begin{aligned}
\delta_{i} & =-\frac{\gamma_{i}^{\prime}\left(\rho_{i}\right)}{\left(\gamma_{i}\left(\rho_{i}\right)-1\right)^{2}}\left[P_{i}+\pi_{i}\left(\rho_{i}\right)\right]+\frac{\gamma_{i}\left(\rho_{i}\right)}{\gamma_{i}\left(\rho_{i}\right)-1} \pi_{i}^{\prime}\left(\rho_{i}\right), \\
\xi_{i} & =\frac{1}{\gamma_{i}\left(\rho_{i}\right)-1} .
\end{aligned}
$$

An explicit expression for $P$ is still available. Introducing $\xi=\sum_{i} z_{i} \xi_{i}$, we find

$$
\rho \varepsilon=\xi P+\sum_{i} z_{i} \gamma_{i}\left(\rho_{i}\right) \pi_{i}\left(\rho_{i}\right) \xi_{i}\left(\rho_{i}\right)
$$

which yields the expression

$$
P\left(\rho_{1}, \rho_{2}, \rho \varepsilon, z\right)=\frac{\rho \varepsilon}{\xi}-\frac{1}{\xi} \sum_{i} z_{i} \gamma_{i}\left(\rho_{i}\right) \pi_{i}\left(\rho_{i}\right) \xi_{i}\left(\rho_{i}\right)=(\gamma-1) \rho \varepsilon-\gamma \pi
$$

with $\xi=1 /(\gamma-1)$ and

$$
\pi=\frac{\gamma-1}{\gamma} \sum_{i} z_{i} \pi_{i}\left(\rho_{i}\right) \frac{\gamma_{i}\left(\rho_{i}\right)-1}{\gamma_{i}\left(\rho_{i}\right)}
$$

Our five-equation model with this equation of state is not equivalent to Shyue's extension [31].

\section{ISOTHERMAL CLOSURE}

\subsection{Hypotheses}

In this section we assume that each fluid $i$ is equipped with an EOS defined in terms of its density $\rho_{i}$ and temperature $T_{i}$, which reads

$$
\varepsilon_{i}:\left(\rho_{i}, T_{i}\right) \mapsto \varepsilon_{i}\left(\rho_{i}, T_{i}\right), \quad P_{i}:\left(\rho_{i}, T_{i}\right) \mapsto P_{i}\left(\rho_{i}, T_{i}\right) .
$$


The isothermal closure reads

$$
T_{1}=T_{2}=T
$$

In the following, we assume that the equations of state satisfy

$$
T_{i}\left(\rho_{i}, \varepsilon_{i}\right) \geq 0 \quad \text { and } \quad T_{i}\left(\rho_{i}, \varepsilon_{i}=0\right)=0, \quad\left(\frac{\partial P_{i}}{\partial \rho_{i}}\right)_{T_{i}}>0, \quad c_{v i}=\left(\frac{\partial \varepsilon_{i}}{\partial T_{i}}\right)_{\rho_{i}}>0
$$

All theses assumptions are physically reasonable although they are not always satisfied. They hold true at least for perfect gases. For a perfect gas EOS, $\left(\partial P_{i} / \partial \rho_{i}\right)_{T_{i}}=c_{v i}$ is a positive constant, so assumption (38) is indeed satisfied. Once again, we emphasize that assumption (38) is just a simple sufficient condition for the five-equation model (with isothermal closure) to be consistent and hyperbolic. Since we shall not use this closure in practice, we did not try to find more general assumptions.

\subsection{Consistency}

The consistency property amounts to being able to compute the global pressure $P$ from the conserved quantities. In the present case, it is equivalent to determining the global temperature $T$, which allows recovery of the partial pressures $P_{i}$ through the EOS and thus the deduction of $P$. We proceed as in the isobaric closure in Section 4.2.

Proposition 5.1. Assumptions (38) and (37) indeed define a single-valued temperature $T$.

Proof. We just need to check that for given positive values of $\rho \varepsilon, \rho_{i}, \rho_{2}$ and $z \in[0,1]$ the system

$$
\left\{\begin{array}{l}
T_{1}\left(\rho_{1}, \varepsilon_{1}\right)-T_{2}\left(\rho_{2}, \varepsilon_{2}\right)=0 \\
z \rho_{1} \varepsilon_{1}+(1-z) \rho_{2} \varepsilon_{2}=\rho \varepsilon
\end{array}\right.
$$

always admits a unique solution $\left(\varepsilon_{1}, \varepsilon_{2}\right)$. We define $\Phi:[0,1] \rightarrow \mathbb{R}$ by

$$
\Phi(x)=T_{1}\left(\rho_{1}, \frac{\rho \varepsilon}{\rho_{1} z} x\right)-T_{2}\left(\rho_{2}, \frac{\rho \varepsilon}{\rho_{2}(1-z)}(1-x)\right) .
$$

Supposing $T_{1}$ and $T_{2}$ to be $\mathcal{C}^{1}$ we obtain by the chain rule

$$
\begin{aligned}
\Phi^{\prime}(x) & =\rho \varepsilon\left\{\frac{1}{\rho_{1} z}\left(\frac{\partial T_{1}}{\partial \varepsilon_{1}}\right)_{\rho_{1}}\left(\rho_{1}, \frac{\rho \varepsilon}{\rho_{1} z} x\right)-\frac{1}{\rho_{2}(1-z)}\left(\frac{\partial T_{2}}{\partial \varepsilon_{2}}\right)_{\rho_{2}}\left(\rho_{2}, \frac{\rho \varepsilon}{\rho_{2}(1-z)}(1-x)\right)\right\} \\
& =\rho \varepsilon\left[\frac{1}{z \rho_{1} c_{v 1}}+\frac{1}{(1-z) \rho_{2} c_{v 2}}\right] .
\end{aligned}
$$

Thus, by hypothesis (38) we have $\Phi^{\prime}(x)>0, \forall x \in[0,1]$. Next, we check that

$$
\Phi(1)=T_{1}\left(\rho_{1}, \frac{\rho \varepsilon}{z \rho_{1}}\right) \geq 0 \quad \text { and } \quad \Phi(0)=-T_{2}\left(\rho_{2}, \frac{\rho \varepsilon}{(1-z) \rho_{2}}\right) \leq 0 .
$$


The smoothness assumptions for $T_{i}$ then provides the existence of $\left.x_{0} \in\right] 0,1[$ such that $\Phi\left(x_{0}\right)=0$. Setting $\varepsilon_{1}=x \rho \varepsilon /\left(z \rho_{1}\right)$ and $\varepsilon_{2}=(1-x) \rho \varepsilon /\left(\rho_{2}(1-z)\right)$ defines a unique solution to our problem.

We end this section by a lemma that provides the expression of the differential for $P$ using the variables $z_{1} \rho_{1}, z_{2} \rho_{2}, \rho u, \rho e, z$.

LEMMA 5.1. The differential of the pressure P for the five-equation model with isothermal closure reads

$$
d P=\frac{1}{\xi} d(\rho \varepsilon)-\sum_{i} \frac{\delta_{i}}{\xi} d\left(z_{i} \rho_{i}\right)+M d z
$$

where the coefficients $\xi, \delta_{i}$, and $M$ are defined by

$$
\begin{aligned}
\rho c_{v} & =\sum_{i} z_{i} \rho_{i} c_{v i}, \\
\frac{1}{\xi} & =\frac{1}{\rho c_{v}} \sum_{i} z_{i}\left(\frac{\partial P_{i}}{\partial T_{i}}\right)_{\rho_{i}} \\
\delta_{i} & =-\xi\left(\frac{\partial P_{i}}{\partial \rho_{i}}\right)_{T_{i}}+h_{i}-\frac{T}{\rho_{i}}\left(\frac{\partial P_{i}}{\partial T_{i}}\right)_{\rho_{i}}, \\
M & =\left[P_{1}-\left(\frac{\partial P_{1}}{\partial \rho_{1}}\right)_{T_{1}}+\frac{\rho_{1}^{2}}{\xi}\left(\frac{\partial \varepsilon_{1}}{\partial \rho_{1}}\right)_{T_{1}}\right]-\left[P_{2}-\left(\frac{\partial P_{2}}{\partial \rho_{2}}\right)_{T_{2}}+\frac{\rho_{2}^{2}}{\xi}\left(\frac{\partial \varepsilon_{2}}{\partial \rho_{2}}\right)_{T_{2}}\right] .
\end{aligned}
$$

Remark. The coefficients $\xi, \delta_{i}$, and $M$ defined by (39) in the context of the isothermal closure are different from those defined in Section 4 in the isobaric case. We choose to give the same names to these different coefficients because it results in the same formulas for the pressure differential and the Jacobian matrix in Section 7.

Proof (Proof of Lemma 5.1). Since $P=\sum_{i} z_{i} P_{i}$ we get

$$
\begin{aligned}
d P & =\sum_{i} P_{i} d z_{i}+\sum_{i} z_{i}\left(\frac{\partial P_{i}}{\partial \rho_{i}}\right)_{T_{i}} d \rho_{i}+\sum_{i} z_{i}\left(\frac{\partial P_{i}}{\partial T_{i}}\right)_{\rho_{i}} d T \\
& =\sum_{i}\left[P_{i}-\rho_{i}\left(\frac{\partial P_{i}}{\partial \rho_{i}}\right)_{T_{i}}\right] d z_{i}+\sum_{i}\left(\frac{\partial P_{i}}{\partial \rho_{i}}\right)_{T_{i}} d\left(z_{i} \rho_{i}\right)+\frac{\rho c_{v}}{\xi} d T .
\end{aligned}
$$

The expression of $d T$ is deduced from the definition of $\rho \varepsilon$ :

$$
d(\rho \varepsilon)=\sum_{i} \varepsilon_{i} d\left(z_{i} \rho_{i}\right)+\sum_{i} z_{i} \rho_{i}\left[\left(\frac{\partial \varepsilon_{i}}{\partial \rho_{i}}\right)_{T_{i}} d \rho_{i}+\left(\frac{\partial \varepsilon_{i}}{\partial T_{i}}\right)_{\rho_{i}} d T\right] .
$$

Using $z_{i} \rho_{i} d \rho_{i}=\rho_{i} d\left(z_{i} \rho_{i}\right)-\rho_{i}^{2} d z_{i}$ and $\rho c_{v}=\sum_{i} z_{i} \rho_{i} c_{v i}$, this yields

$$
d(\rho \varepsilon)=\sum_{i}\left[\varepsilon_{i}+\rho_{i}\left(\frac{\partial \varepsilon_{i}}{\partial \rho_{i}}\right)_{T_{i}}\right] d\left(z_{i} \rho_{i}\right)-\sum_{i} \rho_{i}^{2}\left(\frac{\partial \varepsilon_{i}}{\partial \rho_{i}}\right)_{T_{i}} d z_{i}+\rho c_{v} d T
$$


Now we just have to plug $d T$ from this relation back into relation (40), which gives

$$
d P=\sum_{i}\left[\left(\frac{\partial P_{i}}{\partial \rho_{i}}\right)_{T_{i}}-\frac{1}{\xi}\left(\varepsilon_{i}+\rho_{i}\left(\frac{\partial \varepsilon_{i}}{\partial \rho_{i}}\right)_{T_{i}}\right)\right] d\left(z_{i} \rho_{i}\right)+\frac{d(\rho \varepsilon)}{\xi}+M d z .
$$

To obtain (39) it remains to identify the correct factor in front of $d\left(\rho_{i} z_{i}\right)$,

$$
\left(\frac{\partial P_{i}}{\partial \rho_{i}}\right)_{T_{i}}-\frac{1}{\xi}\left(\varepsilon_{i}+\rho_{i}\left(\frac{\partial \varepsilon_{i}}{\partial \rho_{i}}\right)_{T_{i}}\right)=\frac{1}{\xi}\left[\xi\left(\frac{\partial P_{i}}{\partial \rho_{i}}\right)_{T_{i}}-h_{i}+\frac{T}{\rho_{i}}\left(\frac{\partial P_{i}}{\partial T_{i}}\right)_{\rho_{i}}\right]=-\frac{\delta_{i}}{\xi},
$$

where we used the thermodynamic identity $T\left(\frac{\partial P}{\partial T}\right)_{\rho}=P-\rho^{2}\left(\frac{\partial \varepsilon}{\partial \rho}\right)_{T}$.

\subsection{Hyperbolicity}

By virtue of Proposition 3.1, it is enough to check that the mixture sound velocity $c$ is well defined.

PROPOSITION 5.2. For the five-equation model with isothermal closure, the mixture sound velocity c satisfies

$$
\rho c^{2}=\sum_{i} z_{i} \rho_{i}\left(\frac{\partial P_{i}}{\partial \rho_{i}}\right)_{T_{i}}+\frac{T}{\rho c_{v}}\left[\sum_{i} z_{i}\left(\frac{\partial P_{i}}{\partial T_{i}}\right)_{\rho_{i}}\right]^{2} .
$$

Therefore, under assumption (38) the system is hyperbolic.

Proof. From Lemma 5.1 and the definition of $c^{2}$, we have

$$
\begin{aligned}
\rho c^{2} & =\frac{1}{\xi} \rho h-\sum_{i} \frac{\delta_{i}}{\xi} \rho_{i} z_{i} \\
& =\frac{1}{\xi} \rho h+\sum_{i} \rho_{i} z_{i}\left\{\left(\frac{\partial P_{i}}{\partial \rho_{i}}\right)_{T_{i}}-\frac{h_{i}}{\xi}+\frac{T}{\rho_{i} \xi}\left(\frac{\partial P_{i}}{\partial T_{i}}\right)_{\rho_{i}}\right\} \\
& =\sum_{i} \rho_{i} z_{i}\left(\frac{\partial P_{i}}{\partial \rho_{i}}\right)_{T_{i}}+\frac{T}{\rho c_{v}}\left[\sum_{i} z_{i}\left(\frac{\partial P_{i}}{\partial T_{i}}\right)_{\rho_{i}}\right]^{2},
\end{aligned}
$$

by definition of $\xi$. By assumption, $\left(\partial P_{i} / \partial \rho_{i}\right)_{T_{i}}>0$ for each fluid $i$, and thus $c^{2}$ is positive. The five-equation model with isothermal closure is therefore hyperbolic.

Remark. Formula (41) is reminiscent of the sound velocity $c$ of a pure fluid,

$$
\rho c^{2}=\rho\left(\frac{\partial P}{\partial \rho}\right)_{T}+\frac{T}{\rho c_{v}}\left[\left(\frac{\partial P}{\partial T}\right)_{\rho}\right]^{2} .
$$

However, the sound velocity of the mixture cannot be easily expressed as a combination of the individual sound velocities. 


\subsection{Existence of a Mathematical Entropy}

We recall here Eq. (21), which is formally equivalent to the energy equation

$$
\sum_{i} \rho_{i} z_{i} T_{i} \frac{D S_{i}}{D t}=0
$$

Since $T_{i}=T$, this equation yields

$$
T \sum_{i} \rho_{i} z_{i} \frac{D S_{i}}{D t}=0
$$

Setting $S=\left(\sum_{i} \rho_{i} z_{i} S_{i}\right) / \rho$, we have

$$
\frac{D \rho S}{D t}+\sum_{i} \rho_{i} z_{i} S_{i} \operatorname{divu}=0,
$$

which can be written in conservation form as

$$
\partial_{t} \rho S+\operatorname{div}(\rho S \mathbf{u})=0
$$

This shows that $\rho S$ is a mathematical entropy for the five-equation model with the isothermal closure. However, this entropy is not strictly convex with respect to the variables $z_{i}$.

\subsection{Perfect Gases}

For perfect gases, the isothermal five-equation model reduces to the classical fourequation model introduced in Section 2. Indeed the pressure law then takes the form

$$
P=(\gamma-1) \rho \varepsilon, \quad \text { with } \gamma=\frac{\sum_{i} \rho_{i} z_{i} c_{p_{i}}}{\sum_{i} \rho_{i} z_{i} c_{v i}}
$$

Note that in this case, the computation of the pressure does not require knowledge of the $z_{i}$. The transport equation (10) for $z$ is uncoupled from the rest of the system.

\section{EVOLUTION OF CONSTANT-PRESSURE PROFILES FOR THE ISOBARIC CLOSURE}

Following the lines of [1], we want to ensure that the numerical scheme is able to simulate properly the evolution of a contact discontinuity (or material interface) between the fluids. We thus consider a Riemann problem in which only the volume fraction $z$ is allowed to vary. The exact solution of this problem is the advection of the $z$ profile at constant speed. In this section, we consider numerical schemes based on an approximate Riemann solver which is exact on isolated contact discontinuities. This is true for accurate solvers such as the Roe, Osher, and AUSM schemes, but not for diffusive solvers such as the Lax-Friedrichs, Van Leer, or kinetic schemes. We can state the following proposition. 


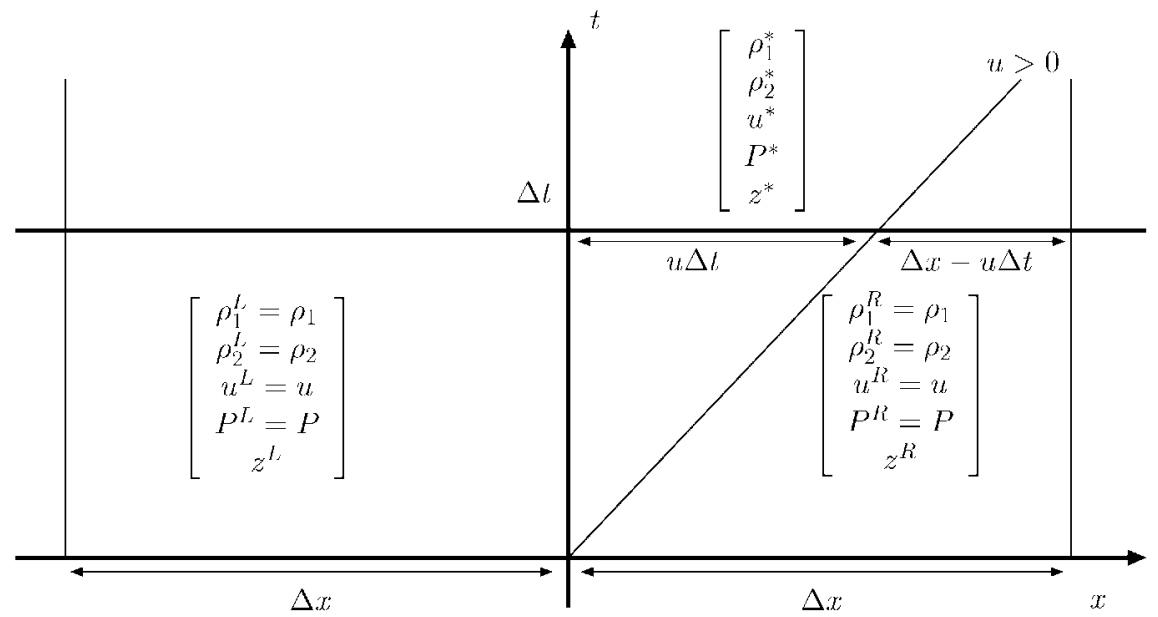

FIG. 1. Constant-pressure profile.

PROPOSITION 6.1. Consider a Riemann problem with initial data

$$
\begin{aligned}
u^{L} & =u^{R}=u, \\
\rho_{i}^{L} & =\rho_{i}^{R}=\rho_{i}, \quad i=1,2, \\
\varepsilon_{i}^{L} & =\varepsilon_{i}^{R}=\varepsilon_{i}, \quad i=1,2, \\
z^{L} & \neq z^{R} .
\end{aligned}
$$

Let $U^{*}$ be the numerical solution of this problem in the cell downstream of the discontinuity, obtained after one time step of a Godunov-type scheme. This solution satisfies

$$
\begin{array}{ll}
u^{*}=u, & \\
\rho_{i}^{*}=\rho_{i}, & i=1,2, \\
\varepsilon_{i}^{*}=\varepsilon_{i}, & i=1,2 .
\end{array}
$$

Remark. Note that although the densities and energies of each phase are the same on each side of the interface $\left(\varepsilon_{i}^{L}=\varepsilon_{i}^{R}\right)$, the density and the energy of the mixture can be different $\left(\rho^{L} \neq \rho^{R}, \varepsilon^{L} \neq \varepsilon^{R}\right)$.

Remark. The property is still valid if either the left or right cell is occupied by a pure fluid $(z=0$ or $z=1)$. Of course, whenever a fluid is not present, its thermodynamic variables are not uniquely defined.

Remark. Because of this property, a contact discontinuity will evolve numerically toward a smeared numerical profile but will still remain at constant pressure and velocity.

Proof. Without restriction we can suppose that $u>0$, so that the downstream state is located in the right cell (see Fig. 1). We denote with superscript $*$ the updated values in this cell. Considering Godunov-type schemes that are exact on isolated contact discontinuities, 
we find that this state is given by

$$
\begin{aligned}
\rho_{i}^{*} z_{i}^{*} & =u \lambda \rho_{i}^{L} z_{i}^{L}+(1-u \lambda) \rho_{i}^{R} z_{i}^{R}, \\
\rho^{*} u^{*} & =u \lambda \rho^{L} u^{L}+(1-u \lambda) \rho^{R} u^{R}, \\
\rho^{*} e^{*} & =u \lambda \rho^{L} e^{L}+(1-u \lambda) \rho^{R} e^{R}, \\
z^{*} & =u \lambda z^{L}+(1-u \lambda) z^{R} .
\end{aligned}
$$

Using the hypothesis on the initial states, one finds that

$$
\begin{aligned}
\rho_{i}^{*} & =\rho_{i}, \\
u^{*} & =u, \\
\rho^{*} \varepsilon^{*} & =u \lambda \rho^{L} \varepsilon^{L}+(1-u \lambda) \rho^{R} \varepsilon^{R} .
\end{aligned}
$$

Next, since $\rho_{i}^{L}=\rho_{i}^{R}$ and $\varepsilon_{i}^{L}=\varepsilon_{i}^{R}$, we get

$$
\rho^{*} \varepsilon^{*}=\sum_{i}\left[u \lambda z_{i}^{L}+(1-u \lambda) z_{i}^{R}\right] \rho_{i} \varepsilon_{i}=\sum_{i} z_{i}^{*} \rho_{i} \varepsilon_{i} .
$$

We remark that $P^{*}=P$ satisfies

$$
\rho^{*} \varepsilon^{*}=\sum_{i} z_{i}^{*}\left(\rho_{i} \varepsilon_{i}\right)\left(P^{*}, \rho_{i}^{*}\right)
$$

Since the equation has a unique solution, then $P^{*}=P$ must be the pressure of the updated right state $(*)$, and $\rho_{i}^{*} \varepsilon_{i}^{*}=\left(\rho_{i} \varepsilon_{i}\right)\left(P^{*}, \rho_{i}^{*}\right)=\rho_{i} \varepsilon_{i}$.

Remark. Following the same lines for the isothermal closure $\left(T_{1}=T_{2}\right)$ leads to $T^{*}=T$, which hence yields $P_{i}^{*}=P_{i}$. However, in general $P^{*}=\sum_{i} z_{i}^{*} P_{i} \neq \sum_{i} z_{i} P_{i}$, so the pressure of the updated right state $(*)$ is different from the initial pressure. The isothermal model therefore does not preserve this type of constant-pressure profile. This is the main reason why we do not use the isothermal closure in numerical computations (see Section 8.2 for a numerical comparison).

\section{NUMERICAL METHOD}

\subsection{Introduction}

In this section, we build a quasi-conservative numerical method for the five-equation model with isobaric closure which is based on a Roe-type scheme [23]. We call it quasiconservative because the five-equation model is not conservative. On the other hand, since the works of Abgrall [1] and Karni [11], it is known that one should give up the conservative character of the numerical method to obtain good stability properties at the material interface. We remark also that, even if the five-equation model were conservative, the complete definition of the usual Roe scheme relies on the linearization of the pressure jump at each cell interface. As is well known, there is no general way to obtain such a linearization when general equations of state are used.

We start from a usual Roe-type scheme. Since some coefficients in the Roe matrix are difficult to determine analytically in the case of real gases, we use a variant of Roe's scheme 
which gives up the usual jump property (see (51)). Instead of requiring a linearization of the pressure jump, this variant relies on the definition of an average sound velocity at each cell interface. Any reasonable averaging procedure can be used for that purpose, making this method very versatile and easy to implement. A similar approach was used in [6] to accommodate general equations of state.

More precisely, assuming that a Roe matrix is well defined, after some algebra our quasi-conservative Roe scheme can be equivalently and conveniently written in terms of a numerical flux which involves only the usual Roe averages for the velocity $u^{*}$, the total enthalpy $H^{*}$, the mass fractions $y_{i}^{*}$, and the averaged speed of sound $c^{*}$. To obtain a simplified scheme (valid for any type of real gas), we then change the definition of $c^{*}$, taken as some explicit average of the speed of sound, rather than enforcing the linearization of the pressure jump.

\subsection{The Roe Scheme}

We work with the set of variables $\mathbf{W}=\left(z_{1} \rho_{1}, z_{2} \rho_{2}, \rho u, \rho e, z\right)$. In this set of variables (as in any other one), the five-equation model (6)-(10) is not in a conservative form. However, it is almost conservative in the sense that the first four equations are conservative.

7.2.1. Jacobian matrix of the five-equation model. We first need to compute the differential of the pressure in terms of the variable W. A straightforward application of Proposition 4.2 provides the following lemma.

LEMMA 7.1. For the isobaric closure the differential of the pressure also reads

$$
d P=\sum_{i} \frac{1}{\xi}\left(\frac{u^{2}}{2}-\delta_{i}\right) d\left(z_{i} \rho_{i}\right)-\frac{u}{\xi} d(\rho u)+\frac{1}{\xi} d(\rho e)+M d z
$$

By introducing the coefficients $\beta_{i}=\left(u^{2} / 2-\delta_{i}\right) / \xi$, an easy computation yields the quasilinear form of system (6)-(10) with isobaric closure.

PROPOSITION 7.1. For smooth solutions, the five-equation system with isobaric closure is equivalent to the system

$$
\frac{\partial \mathbf{W}}{\partial t}+A(\mathbf{W}) \frac{\partial \mathbf{W}}{\partial x}=0
$$

where the matrix A (called, by a slight abuse of language, the Jacobian matrix of the system) is given by

$$
A(\mathbf{W})=\left(\begin{array}{ccccc}
u y_{2} & -u y_{1} & y_{1} & 0 & 0 \\
-u y_{2} & u y_{1} & y_{2} & 0 & 0 \\
\left(\beta_{1}-u^{2}\right) & \left(\beta_{2}-u^{2}\right) & \left(2-\frac{1}{\xi}\right) u & \frac{1}{\xi} & M \\
u\left(\beta_{1}-H\right) & u\left(\beta_{2}-H\right) & \left(H-\frac{u^{2}}{\xi}\right) & \left(1+\frac{1}{\xi}\right) u & u M \\
0 & 0 & 0 & 0 & u
\end{array}\right) .
$$

We now give the eigenelements of the Jacobian matrix. 
Proposition 7.2. The Jacobian matrix $A(\mathbf{W})$ is diagonalizable with eigenvalues $\lambda_{i}(\mathbf{W})$, right eigenvectors $\mathbf{r}_{i}(\mathbf{W})$, and left eigenvectors $\mathbf{l}_{i}(\mathbf{W})$ given by

$$
\begin{aligned}
& \lambda_{1}(\mathbf{W})=u-c, \quad \lambda_{2}(\mathbf{W})=\lambda_{3}(\mathbf{W})=\lambda_{4}(\mathbf{W})=u, \quad \lambda_{5}(\mathbf{W})=u+c, \\
& \mathbf{r}_{1}(\mathbf{W})=\left[\begin{array}{c}
y_{1} \\
y_{2} \\
u-c \\
H-u c \\
0
\end{array}\right], \quad \mathbf{r}_{2}(\mathbf{W})=\left[\begin{array}{c}
1 \\
0 \\
u \\
\frac{u^{2}}{2}+\delta_{1} \\
0
\end{array}\right], \quad \mathbf{r}_{3}(\mathbf{W})=\left[\begin{array}{c}
0 \\
1 \\
u \\
\frac{u^{2}}{2}+\delta_{2} \\
0
\end{array}\right], \\
& \mathbf{r}_{4}(\mathbf{W})=\left[\begin{array}{c}
0 \\
0 \\
0 \\
-M \xi \\
1
\end{array}\right], \quad \mathbf{r}_{5}(\mathbf{W})=\left[\begin{array}{c}
y_{1} \\
y_{2} \\
u+c \\
H+u c \\
0
\end{array}\right] \\
& \mathbf{l}_{1}(\mathbf{W})=\frac{1}{2 c^{2}}\left[\begin{array}{c}
\beta_{1}+u c \\
\beta_{2}+u c \\
-c-\frac{u}{\xi} \\
\frac{1}{\xi} \\
M
\end{array}\right], \quad \mathbf{l}_{2}(\mathbf{W})=\frac{1}{\xi c^{2}}\left[\begin{array}{c}
\xi c^{2}+y_{1}\left(\delta_{1}-\frac{u^{2}}{2}\right) \\
y_{1}\left(\delta_{2}-\frac{u^{2}}{2}\right) \\
u y_{1} \\
-y_{1} \\
-y_{1} M \xi
\end{array}\right] \\
& \mathbf{l}_{3}(\mathbf{W})=\frac{1}{\xi c^{2}}\left[\begin{array}{c}
y_{2}\left(\delta_{1}-\frac{u^{2}}{2}\right) \\
\xi c^{2}+y_{2}\left(\delta_{2}-\frac{u^{2}}{2}\right) \\
u y_{2} \\
-y_{2} \\
-y_{2} M \xi
\end{array}\right], \quad \mathbf{l}_{4}(\mathbf{W})=\left[\begin{array}{l}
0 \\
0 \\
0 \\
0 \\
1
\end{array}\right], \quad \mathbf{l}_{5}(\mathbf{W})=\frac{1}{2 c^{2}}\left[\begin{array}{c}
\beta_{1}-u c \\
\beta_{2}-u c \\
c-\frac{u}{\xi} \\
\frac{1}{\xi} \\
M
\end{array}\right] \text {. }
\end{aligned}
$$

7.2.2. Roe matrix. We now define a Roe-type linearization for the five-equation model with isobaric closure. Following Roe's approach [23], we consider a 1-D Riemann problem with piecewise-constant initial data $\mathbf{W}_{L}$ (left) and $\mathbf{W}_{R}$ (right). The goal is to define a Roe matrix $A^{*}$ which is a linearization of the Jacobian matrix satisfying some additional properties. We first recall some classical notation associated with the average Roe matrix definition: Let $a$ be any vector or scalar quantity; we note $\Delta a=a_{R}-a_{L}$ and

$$
\bar{a}=\frac{\sqrt{\rho_{L}} a_{L}+\sqrt{\rho_{R}} a_{R}}{\sqrt{\rho_{L}}+\sqrt{\rho_{R}}}, \quad \underline{a}=\frac{\sqrt{\rho_{L}} a_{R}+\sqrt{\rho_{R}} a_{L}}{\sqrt{\rho_{L}}+\sqrt{\rho_{R}}} .
$$

Since the five-equation model is not in conservative form, one cannot impose the usual jump condition. Rather, $A^{*}$ must satisfy the weak form of Roe's jump condition,

$$
\left[\begin{array}{c}
\Delta\left(z_{1} \rho_{1} u\right) \\
\Delta\left(z_{2} \rho_{2} u\right) \\
\Delta\left(\rho u^{2}+P\right) \\
\Delta(\rho H u) \\
u^{*} \Delta(z)
\end{array}\right]=A^{*}\left(\mathbf{W}_{R}-\mathbf{W}_{L}\right)
$$


where $u^{*}$ is a properly chosen average value of $u$, and $A^{*}$ must have the same structure as the Jacobian matrix (49), namely,

$$
A^{*}=\left(\begin{array}{ccccc}
u^{*} y_{2}^{*} & -u^{*} y_{1}^{*} & y_{1}^{*} & 0 & 0 \\
-u^{*} y_{2}^{*} & u^{*} y_{1}^{*} & y_{2}^{*} & 0 & 0 \\
\left(\beta_{1}^{*}-\left(u^{*}\right)^{2}\right) & \left(\beta_{2}^{*}-\left(u^{*}\right)^{2}\right) & \left(2-\frac{1}{\xi^{*}}\right) u^{*} & \frac{1}{\xi^{*}} & M^{*} \\
u^{*}\left(\beta_{1}^{*}-H^{*}\right) & u^{*}\left(\beta_{2}^{*}-H^{*}\right) & \left(H^{*}-\frac{\left(u^{*}\right)^{2}}{\xi^{*}}\right) & \left(1+\frac{1}{\xi^{*}}\right) u^{*} & u^{*} M^{*} \\
0 & 0 & 0 & 0 & u^{*}
\end{array}\right),
$$

with $\beta_{i}^{*}=\left[\left(u^{*}\right)^{2} / 2-\delta_{i}^{*}\right] / \xi^{*}$. This last property ensures that $A^{*}$ is diagonalizable with eigenelements given by Proposition 7.2. We choose $u^{*}$ to be the usual Roe average

$$
u^{*}=\bar{u}
$$

Then, after some easy algebra, (51) implies that $H$ and $y_{i}$ are also Roe averages:

$$
y_{i}^{*}=\overline{y_{i}}, \quad H^{*}=\bar{H} .
$$

Finally, the jump condition (51) is satisfied if there exist averaged partial derivatives of the equation of state $\xi^{*}, \delta_{i}^{*}$, and $M^{*}$ such that

$$
\Delta(\rho \varepsilon)=\xi^{*} \Delta P-\xi^{*} M^{*} \Delta z+\sum_{i} \delta_{i}^{*} \Delta\left(z_{i} \rho_{i}\right)
$$

For perfect gases, one can explicitly find the values of $\xi^{*}, \delta_{i}^{*}$, and $M^{*}$. However, for real gases this is not the case and we shall assume the existence of these coefficients in the pressure linearization (54).

Remark. The matrix $A^{*}$ thus defined is a quasi-conservative Roe matrix for the fiveequation system. Since it has the same structure as the Jacobian matrix, Proposition 7.2 delivers its eigenvalues and eigenvectors in terms of a coefficient $c^{*}$ defined by

$$
\left(c^{*}\right)^{2}=\left(\bar{H}-\frac{\bar{u}^{2}}{2}+\sum_{i} \overline{y_{i}} \delta_{i}^{*}\right) / \xi^{*} .
$$

We call $c^{*}$ the sound velocity of the matrix $A^{*}$. Note that $A^{*}$ is not defined as the Jacobian matrix $A\left(\mathbf{W}^{*}\right)$ of some average state $\mathbf{W}^{*}$, and thus $c^{*}$ is not the true sound velocity of a physical state.

7.2.3. Numerical scheme. With the knowledge of the Roe matrix $A^{*}$, we next define our first-order quasi-conservative Roe scheme. Introducing the subset of the four conservative variables $\mathbf{V}=\left(z_{1} \rho_{1}, z_{2} \rho_{2}, \rho u, \rho e\right)$, we see that this scheme is designed to be conservative for $\mathbf{V}$ and nonconservative for $z$ (recall that $\mathbf{W}=(\mathbf{V}, z)$ ). Actually, the last advection equation for $z$ is decoupled from the first four ones.

Recall that the dissipative part of the usual Roe numerical flux is given by $\left|A^{*}\right| \Delta \mathbf{W}$ with $\Delta \mathbf{W}=\left(\Delta\left(z_{1} \rho_{1}\right), \Delta\left(z_{2} \rho_{2}\right), \Delta(\rho u), \Delta(\rho e), \Delta z\right)$. We only keep its first four components 
denoted by $\mathbf{R}\left(\mathbf{W}_{L}, \mathbf{W}_{R}\right)$. More precisely, for $1 \leq k \leq 4$, the $k$ th component of $\mathbf{R}$ is

$$
\mathbf{R}_{k}=\left(\left|A^{*}\right| \Delta \mathbf{W}\right)_{k}=\sum_{j=1}^{5}\left|\lambda_{j}\right| \alpha_{j}\left(\mathbf{r}_{\mathbf{j}}\right)_{k},
$$

with $\alpha_{j}=\mathbf{l}_{\mathbf{j}} \cdot \Delta \mathbf{W}$. Introducing the conservative flux for the four first equations,

$$
\mathbf{f}(\mathbf{W})=\left(z_{1} \rho_{1} u, z_{2} \rho_{2} u, \rho u^{2}+P, \rho H u\right)
$$

we divide the scheme into two parts:

- The conservative variables $\mathbf{V}$ are updated by

$$
\mathbf{V}_{i}^{n+1}=\mathbf{V}_{i}^{n}-\frac{\Delta t}{\Delta x}\left[\mathbf{G}_{i+1 / 2}^{n}-\mathbf{G}_{i-1 / 2}^{n}\right]
$$

with the numerical flux

$$
\mathbf{G}_{i+1 / 2}^{n}=\frac{1}{2}\left[f\left(\mathbf{W}_{i}\right)+f\left(\mathbf{W}_{i+1}\right)\right]-\frac{1}{2} \mathbf{R}\left(\mathbf{W}_{i}, \mathbf{W}_{i+1}\right), \quad \text { and }
$$

- the color function $z$ is advected using the upwind scheme

$$
z_{i}^{n+1}=z_{i}^{n}-\frac{\Delta t}{2 \Delta x}\left[\left(\bar{u}_{i+1 / 2}\right)^{-} \Delta z_{i+1 / 2}+\left(\bar{u}_{i-1 / 2}\right)^{+} \Delta z_{i-1 / 2}\right]
$$

where $\Delta z_{i+1 / 2}=z_{i+1}-z_{i}, \bar{u}_{i+1 / 2}$ is the Roe average of $u$ between the cells $i$ and $i+1$, and $u^{ \pm}=(u \pm|u|) / 2$.

A priori, this definition of the scheme requires knowledge of the coefficients $\xi^{*}, \delta_{i}^{*}$, and $M^{*}$ since $A^{*}$ depends on their values. Nevertheless, it is possible to write $\mathbf{R}$ in such a way that these coefficients cancel out and only the average sound velocity $c^{*}$ remains. Let us explain this algebraic fact. By virtue of Lemma 7.1 the coefficients $\alpha_{j}$ can be written as

$$
\begin{aligned}
& \alpha_{1}=\frac{1}{2 c^{* 2}}\left(\Delta P-\underline{\rho} c^{*} \Delta u\right), \\
& \alpha_{2}=\Delta\left(z_{1} \rho_{1}\right)-\frac{\overline{y_{1}}}{c^{* 2}} \Delta P, \\
& \alpha_{3}=\Delta\left(z_{2} \rho_{2}\right)-\frac{\overline{y_{2}}}{c^{* 2}} \Delta P, \\
& \alpha_{4}=\Delta z, \\
& \alpha_{5}=\frac{1}{2 c^{* 2}}\left(\Delta P+\underline{\rho} c^{*} \Delta u\right) .
\end{aligned}
$$

The coefficients $\delta_{i}^{*}$ and $M^{*}$ only appear in the right eigenvectors $\mathbf{r}_{j}$ for $j=2$ through 4 . We have

$$
\sum_{j=2}^{4}\left|\lambda_{j}\right| \alpha_{j} \mathbf{r}_{j}=|u|\left[\begin{array}{c}
\alpha_{2} \\
\alpha_{3} \\
\bar{u}\left(\alpha_{2}+\alpha_{3}\right) \\
B \\
\alpha_{4}
\end{array}\right]
$$


with $B=\frac{\bar{u}^{2}}{2}\left[\alpha_{2}+\alpha_{3}\right]+\alpha_{2} \delta_{1}^{*}+\alpha_{3} \delta_{2}^{*}-\alpha_{4} M^{*} \xi^{*}$. Using the values of $\alpha_{2}, \alpha_{3}$, and $\alpha_{4}$ from Eqs. (58)-(60), we get

$$
B=\frac{\bar{u}^{2}}{2}\left[\Delta \rho-\frac{\Delta P}{\left(c^{*}\right)^{2}}\right]-M^{*} \xi^{*} \Delta z+\sum_{i=1}^{2}\left(\Delta\left(\rho_{i} z_{i}\right)-\frac{\overline{y_{i}}}{\left(c^{*}\right)^{2}} \Delta P\right) \delta_{i}^{*} .
$$

In view of the jump relation (54) and definition (55) of $c^{*}$ we deduce

$$
B=\frac{\bar{u}^{2}}{2} \Delta \rho+\Delta(\rho \varepsilon)-\frac{\bar{H}}{c^{* 2}} \Delta P,
$$

and (62) relies only on the average speed of sound $c^{*}$. To sum up, $\mathbf{R}$ can be written as

$$
\mathbf{R}\left(\mathbf{W}_{L}, \mathbf{W}_{R}\right)=\left|\bar{u}-c^{*}\right| \alpha_{1}\left[\begin{array}{c}
\overline{y_{1}} \\
\overline{y_{2}} \\
\bar{u}-c^{*} \\
\bar{H}-\bar{u} c^{*}
\end{array}\right]+|\bar{u}|\left[\begin{array}{c}
\alpha_{2} \\
\alpha_{3} \\
\bar{u}\left(\alpha_{2}+\alpha_{3}\right) \\
B
\end{array}\right]+\left|\bar{u}+c^{*}\right| \alpha_{5}\left[\begin{array}{c}
\overline{y_{1}} \\
\overline{y_{2}} \\
\bar{u}+c^{*} \\
\bar{H}+\bar{u} c^{*}
\end{array}\right],
$$

with the coefficients $\alpha_{1}-\alpha_{5}$ and $B$ defined by (57)-(61) and (62).

\subsection{The Simplified Solver}

Eventually, to derive a simplified numerical scheme, we simply replace the exact formula (55) for the average sound velocity $c^{*}$ (which requires knowledge of $\delta_{i}^{*}$ and $\xi^{*}$ ) by another simpler and explicit formula. The main idea, as in [6], is to compute $c^{*}$ directly as an average value of the mixture sound velocity. Many reasonable averages are available for that purpose. For instance, $c^{*}=\bar{c}$, or $\left(c^{*}\right)^{2}=\left(\bar{H}-\frac{\bar{u}^{2}}{2}+\sum_{i} \overline{y_{i}} \overline{\delta_{i}}\right) / \bar{\xi}$.

For the isobaric closure, it is convenient to use instead formula (29), i.e.,

$$
\left(c^{*}\right)^{2}=\frac{\sum_{i} \overline{y_{i} \xi_{i} c_{i}^{2}}}{\bar{\xi}} .
$$

This is the choice we have used in the numerical applications. For more details on the computation of $\xi$ and $c$ for van der Waals, see, for instance, [12].

Note that it might be impossible to enforce the jump condition (54) with the choice $\xi^{*}=\bar{\xi}$ and $\delta_{i}^{*}=\overline{\delta_{i}}$. In this sense, our numerical scheme differs from the original Roe scheme.

Finally, we use the entropy fix of Harten and Hyman [9] in conjunction with our Roe-type scheme.

\subsection{Second-Order Extensions}

7.4.1. Second order in space. Second-order accuracy is reached using a MUSCL method. Slopes are defined for following variables $z_{1} \rho_{1}, z_{2} \rho_{2}, u, P, z$ with a minmod limiter. At each cell interface two states $\mathbf{V}_{i+1 / 2}^{R, L}$ are reconstructed, and the numerical flux reads

$$
\left\{\begin{array}{l}
\mathbf{V}_{i}^{n+1}=\mathbf{V}_{i}^{n}-\frac{\Delta t}{\Delta x}\left[\mathbf{G}_{i+1 / 2}^{n}-\mathbf{G}_{i-1 / 2}^{n}\right] \\
\mathbf{G}_{i+1 / 2}^{n}=\mathbf{G}\left(\mathbf{W}_{i+1 / 2}^{L}, \mathbf{W}_{i+1 / 2}^{R}\right)
\end{array}\right.
$$

with $\mathbf{W}=(\mathbf{V}, z)$ and $\mathbf{G}(\cdot, \cdot)$ defined in Section 7.2.3. 
Let us detail the construction of the states $\mathbf{V}_{i+1 / 2}^{R, L}$. For each flow parameter $a$ we define two states $a_{i+1 / 2}^{R}$ and $a_{i+1 / 2}^{R}$ on each side of the cell interface $(i+1 / 2)$ by

$$
\begin{aligned}
& a_{i+1 / 2}^{R}=a_{i+1}-\frac{1}{2} \operatorname{minmod}\left(\Delta a_{i+3 / 2}, \Delta a_{i+1 / 2}\right), \\
& a_{i+1 / 2}^{L}=a_{i}+\frac{1}{2} \operatorname{minmod}\left(\Delta a_{i+1 / 2}, \Delta a_{i-1 / 2}\right),
\end{aligned}
$$

with

$$
\operatorname{minmod}(x, y)=\operatorname{sign}(x) \max \{0, \min [|x|, y \operatorname{sign}(x)]\} .
$$

This reconstruction process is applied to the primitive variables $\left(z_{1} \rho_{1}, z_{2} \rho_{2}, u, P, z\right)$, which yields the values of the reconstructed conservative variables at the cell interface $\mathbf{W}_{i+1 / 2}^{R, L}$.

Special care is needed for the numerical scheme advecting the $z$ variable. As discussed in Section 6, the second-order advection numerical scheme for $z$ must be compatible with relation (46), namely,

$$
z^{*}=\lambda u z^{L}+(1-\lambda u) z^{R}
$$

in regions where the solution is a constant-pressure profile as described in Section 6. We propose two numerical schemes that satisfy this criterion. We set

$$
\hat{\Delta} a_{i+1 / 2}=a_{i+1 / 2}^{R}-a_{i+1 / 2}^{L},
$$

with $a$ being a flow variable.

The first scheme is the one proposed by Abgrall for his quasi-conservative method in [1], namely,

$$
z_{i}^{n+1}-z_{i}^{n}+\frac{\Delta t}{2 \Delta x}\left[u_{i}^{n} \hat{\Delta} z_{i+1 / 2}+u_{i}^{n} \hat{\Delta} z_{i-1 / 2}-\left|u_{i}^{n}\right| \hat{\Delta} z_{i+1 / 2}+\left|u_{i}^{n}\right| \hat{\Delta} z_{i-1 / 2}\right]=0
$$

We found a second scheme that provides good results too. The idea is to write the transport equation for $z$ as

$$
\frac{\partial z}{\partial t}+\operatorname{div}(z \mathbf{u})-z \operatorname{div}(\mathbf{u})=0
$$

and to apply a second-order scheme for the conservative part of (67) together with a nonconservative correction for the last term of (67). The resulting scheme reads

$$
\begin{aligned}
z_{i}^{n+1} & =z_{i}^{n}-\lambda\left(q_{i+1 / 2}-q_{i-1 / 2}\right)+\lambda z_{i}\left(r_{i+1 / 2}-r_{i-1 / 2}\right), \\
q_{i+1 / 2} & =q\left(\mathbf{W}_{i+1 / 2}^{L}, \mathbf{W}_{i+1 / 2}^{R}\right), \\
q\left(\mathbf{W}_{L}, \mathbf{W}_{R}\right) & =\frac{1}{2}\left(u_{R} z_{R}+u_{L} z_{L}\right)-\frac{1}{2}\left|\frac{u_{R}+u_{L}}{2}\right|\left(z_{R}-z_{L}\right), \\
r_{i+1 / 2} & =\frac{u_{i+1 / 2}^{R}+u_{i+1 / 2}^{L}}{2} .
\end{aligned}
$$


7.4.2. Second order in time. Second-order accuracy in time is obtained by using a twopoint Runge-Kutta method applied to the full five-equation solver. Let $\mathbf{W}^{n}$ be the set of flow variables $\left(\rho_{1} z_{1}, \rho_{2} z_{2}, \rho u, \rho e, z\right)$ at the time step $n$. We denote by $\mathbf{L}$ the discrete operator associated with the first-order in time solver such that the previous algorithm reads

$$
\mathbf{W}^{n+1}=\mathbf{W}^{n}+\Delta t \mathbf{L}\left[\mathbf{W}^{n}\right]
$$

With this notation the second-order Runge-Kutta method is simply

$$
\begin{aligned}
\mathbf{W}^{n+1 / 2} & =\mathbf{W}^{n}+\Delta t \mathbf{L}\left[\mathbf{W}^{n}\right], \\
\mathbf{W}^{n+1} & =\frac{\mathbf{W}^{n}+\mathbf{W}^{n+1 / 2}}{2}+\frac{\Delta t}{2} \mathbf{L}\left[\mathbf{W}^{n+1 / 2}\right] .
\end{aligned}
$$

\section{NUMERICAL TESTS}

We present a selection of numerical tests performed with the five-equation model, using our second-order algorithm, in both time and space, as described in Section 7.4. The two proposed schemes (66) or (68)-(71) for the advection of the color function $z$ give the same results except as otherwise mentioned. The CFL number is always equal to $1 / 2$. We use the isobaric closure except as otherwise mentioned. More examples, as well as comparisons with other models, are available in [12].

\subsection{Pure Interface Advection}

The first test is a 1-D interface advection between two Mie-Gruneisen fluids (see Section 8.1). The length of the computational domain is $1.0 \mathrm{~m}$, and initially the interface is located at $x=0.5 \mathrm{~m}$. Each Mie-Gruneisen material has a special type of EOS, introduced by Cochran and Chan as

$$
P=\bar{P}(\rho)+(\gamma-1)[\rho \varepsilon-\rho \bar{\varepsilon}(\rho)]
$$

with

$$
\left\{\begin{array}{l}
\bar{P}(\rho)=A_{1}\left(\frac{\rho_{0}}{\rho}\right)^{-E_{1}}-A_{2}\left(\frac{\rho_{0}}{\rho}\right)^{-E_{2}}, \\
\bar{\varepsilon}(\rho)=-\frac{A_{1}}{\rho_{0}\left(1-E_{1}\right)}\left[\left(\frac{\rho_{0}}{\rho}\right)^{1-E_{1}}-1\right]+\frac{A_{2}}{\rho_{0}\left(1-E_{2}\right)}\left[\left(\frac{\rho_{0}}{\rho}\right)^{1-E_{2}}-1\right]-c_{v} T_{0} .
\end{array}\right.
$$

The values of the constants $\gamma, \rho_{0}, T_{0}, c_{v}, A_{1}, A_{2}, E_{1}, E_{2}$ for both gases are shown in Table II (taken from [25]). The initial density of the fluid on the left side of the domain is $\rho=9000 \mathrm{~kg} \cdot \mathrm{m}^{-3}$ and that of the fluid on the right side is $\rho=2000 \mathrm{~kg} \cdot \mathrm{m}^{-3}$. Both fluids have the same pressure $P=10^{5} \mathrm{~Pa}$ and velocity $u=1500 \mathrm{~m} \cdot \mathrm{s}^{-1}$ at $t=0$.

The discretization is done on a 100-cell grid. Boundary conditions are constant states on both right and left sides of the domain. Figure 2 displays the numerical solution of the five-equation model obtained at $t=240 \mu$ s after 269 time steps (the solid line is the exact solution and the dotted line is the numerical solution). This test shows the perfect behavior of the model as far as preserving the constant-velocity and -pressure profile is concerned. Similar tests have been successfully performed for other EOSs, including stiffened gas and van der Waals fluid (as in [30]). 
TABLE II

Mie-Gruneisen Material Properties

\begin{tabular}{lcc}
\hline \multicolumn{1}{c}{ Parameter } & Left material & Right material \\
\hline$\gamma$ & 3 & 1.93 \\
$c_{v}\left(\mathrm{~J} \cdot \mathrm{kg}^{-1} \cdot \mathrm{K}^{-1}\right)$ & 393 & 1087 \\
$A_{1}(\mathrm{~Pa})$ & $1.45667 \times 10^{11}$ & $1.2871 \times 10^{10}$ \\
$A_{2}(\mathrm{~Pa})$ & $1.47751 \times 10^{11}$ & $1.34253 \times 10^{10}$ \\
$E_{1}$ & 2.994 & 4.1 \\
$E_{2}$ & 1.994 & 3.1 \\
$T_{0}(\mathrm{~K})$ & 300 & 300 \\
$\rho_{0}\left(\mathrm{~kg} \cdot \mathrm{m}^{-3}\right)$ & 8900 & 1840 \\
\hline
\end{tabular}

Color function $z$

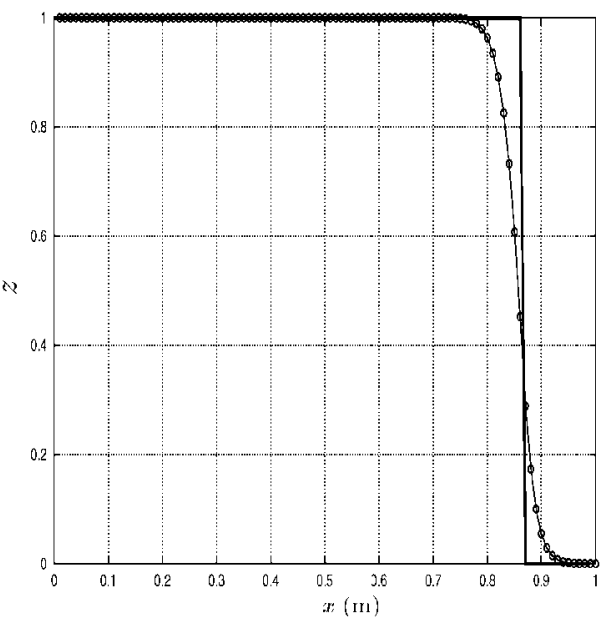

Pressure

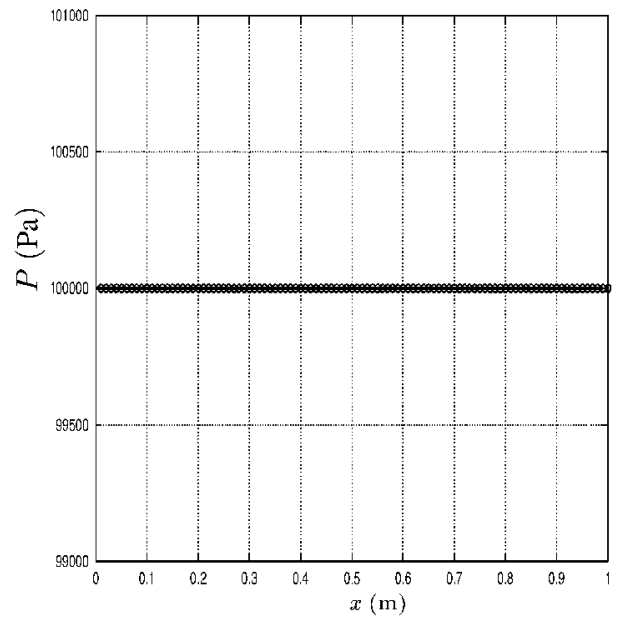

Density

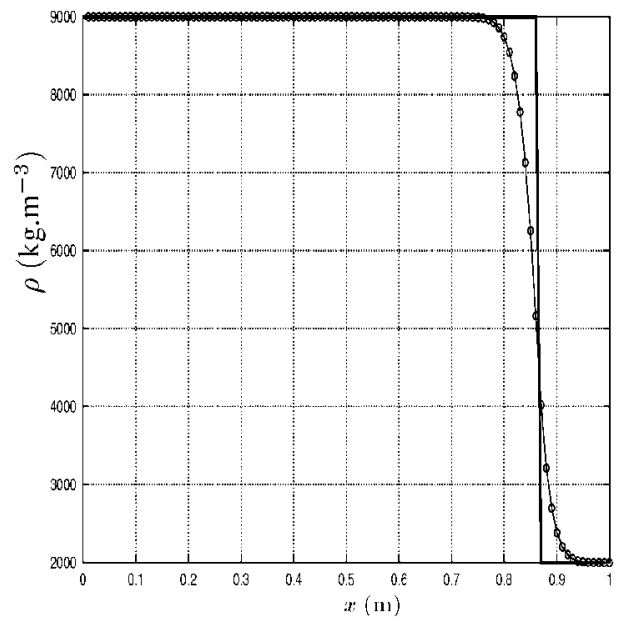

Velocity

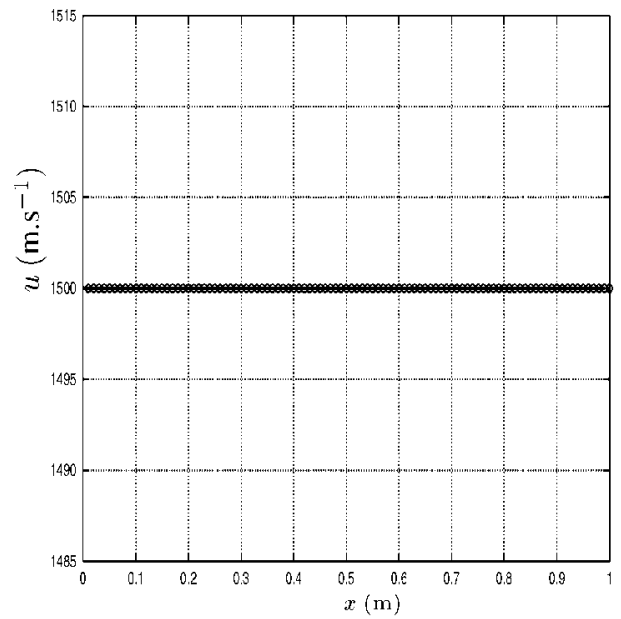

FIG. 2. Interface advection problem between two Mie-Gruneisen materials: exact (solid line) and numerical (dotted line) results. 
The interface is spread over roughly 15 cells due to numerical diffusion. Of course, it is possible to sharpen the advection front of the volume fraction $z$, but, since this is a separate issue, we do not discuss this problem here. Let us simply mention that we tested two classical methods: First, we add antidiffusive terms in the transport equation for $z$ (see [13]); second, we reinitialize a sharp $z$ profile by using a level set method (see [12]). There are other possible approaches, including the use of antidiffusive schemes for the transport equation [7].

\subsection{Comparison of the Isobaric and Isothermal Closures: Interface Advection}

We perform a simple interface advection which confirms that only the isobaric closure preserves constant-pressure profiles. Even for perfect gases, the isothermal closure is not stable at material interfaces. The two perfect gases, lying in a 1-m tube, are initially separated by an interface located at $x=0.5 \mathrm{~m}$ and share the same pressure $P=10^{5} \mathrm{~Pa}$ and the same velocity $u=10^{3} \mathrm{~m} \cdot \mathrm{s}^{-1}$. On the left side the adiabatic exponent is $\gamma=1.4$, the specific heat at constant volume is $c_{v}=732 \mathrm{~J} \cdot \mathrm{kg}^{-1} \cdot \mathrm{K}^{-1}$, and the density is $\rho=50 \mathrm{~kg} \cdot \mathrm{m}^{-3}$, while on the right side we have $\gamma=4.4, c_{v}=1000 \mathrm{~J} \cdot \mathrm{kg}^{-1} \cdot \mathrm{K}^{-1}$, and $\rho=10^{3} \mathrm{~kg} \cdot \mathrm{m}^{-3}$.

The domain is discretized with 100 cells and the boundary conditions are constant states on both sides. Figure 3 gives the profiles of the flow variables at $t=50 \mu$ s obtained with the two (isobaric and isothermal) solvers at second order in space and time. While the pressure and the velocity are correctly preserved by the isobaric closure, there are strong overshoots and undershoots or the isothermal closure.

\subsection{Shock Tube}

Our third test (originating from [30]) is a classical 1-D shock tube problem. It involves a stiffened gas modeling a liquid on the left side of the domain and a van der Waals gas on the right. The domain is $1-\mathrm{m}$ long. The interface is initially located at $x=0.7 \mathrm{~m}$. The EOS of the van der Waals gas is

$$
\left\{\begin{array}{l}
P=\left(\frac{\gamma-1}{1-b \rho}\right)\left(\rho \varepsilon-a \rho^{2}\right)-a \rho^{2}, \\
\gamma=1.4, \quad a=5, \quad b=10^{-3},
\end{array}\right.
$$

while that of the stiffened gas is

$$
\left\{\begin{array}{l}
P=(\gamma-1) \rho \varepsilon-\gamma \pi, \\
\gamma=4.4, \quad \pi=6 \times 10^{8} \mathrm{~Pa} .
\end{array}\right.
$$

At $t=0$ both fluids are at rest, while the pressure and the density are $P=10^{9} \mathrm{~Pa}, \rho=$ $10^{3} \mathrm{~kg} \cdot \mathrm{m}^{-3}$ on the left side of the domain and $P=10^{5} \mathrm{~Pa}, \rho=50 \mathrm{~kg} \cdot \mathrm{m}^{-3}$ on the right side.

Constant boundary conditions are applied on both sides of the domain discretized with 300 cells. Figure 4 displays the numerical solution (dotted line) of our five-equation solver obtained at $t=240 \mu \mathrm{s}$, which is compared to a converged solution (solid line) obtained with 3000 cells. Our results are also in very good agreement with the solution obtained in [30] (with a different model). 
Color function $z$

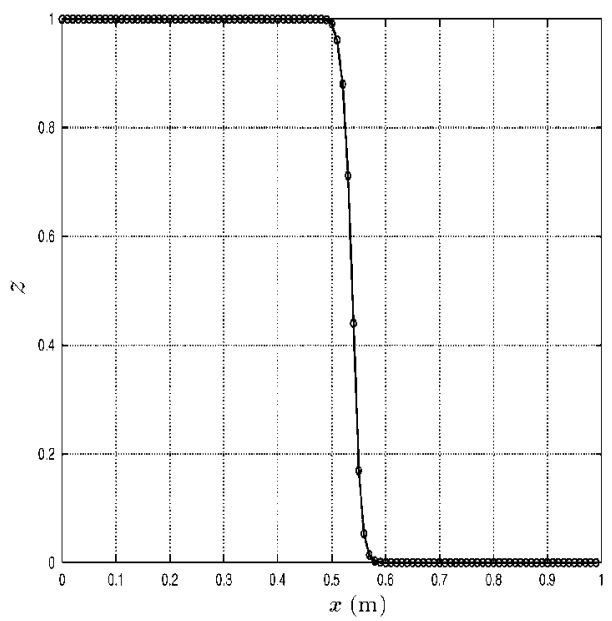

Pressure

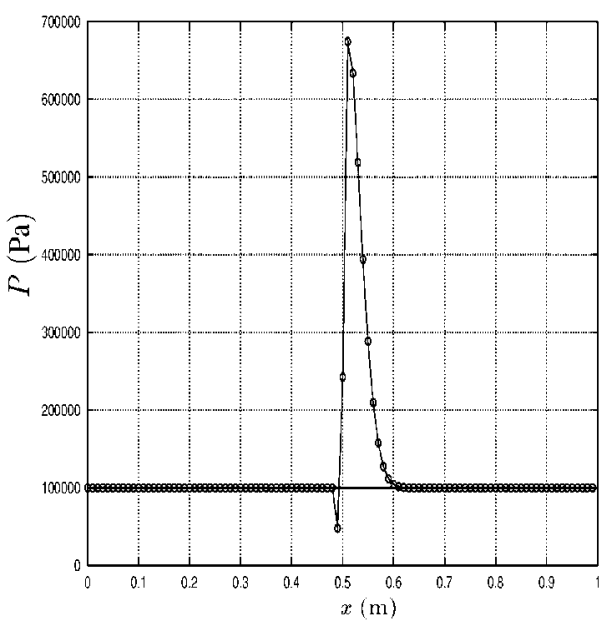

Density

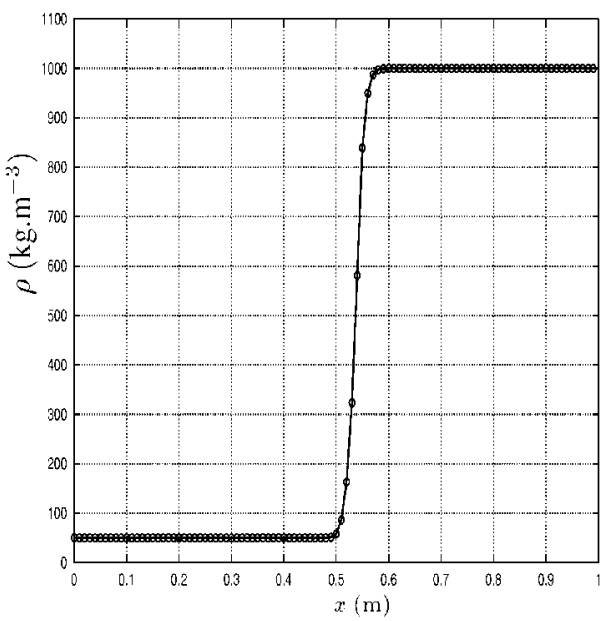

Velocity

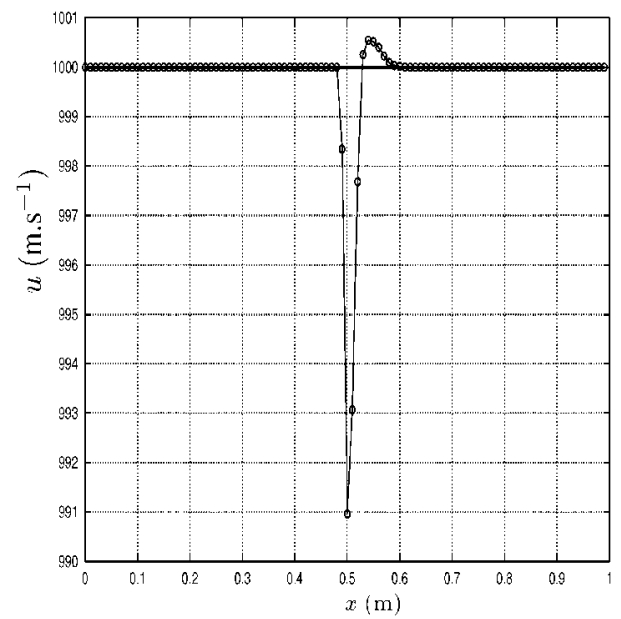

FIG. 3. Advection test between two perfect gases: comparison between the isobaric (thick solid line) and the isothermal (thin dotted line) closure for the five-equation model.

\subsection{Interface-Shock Interaction}

The fourth test (again from [30]) is more delicate. It is a 1-D shock-interface interaction problem involving a stiffened gas and a van der Waals gas. The domain is 1-m long and is discretized with 400 cells. A van der Waals gas is on the left side and a stiffened gas is on the right. The interface between the fluids is initially located at $x=0.4 \mathrm{~m}$. The EOSs used for this test are provided as in the previous examples by relations (72) and (73).

A 1.422 Mach shock travels from the right to the left. Initially the shock wave is in the pure stiffened gas area at $x=0.5 \mathrm{~m}$. The initial fluid variables are recalled in Table III.

The initial pressure ratio between the preshock and the postshock states is $10^{4}$, which indicates a very strong shock. The flow variables are displayed at $t=270 \mu \mathrm{s}$ in Fig. 5 with a refined solution obtained with a 3000-cell grid. The good profile of the solution obtained here can be checked by comparison with [30]. This test is more delicate than the previous 
Color function $z$

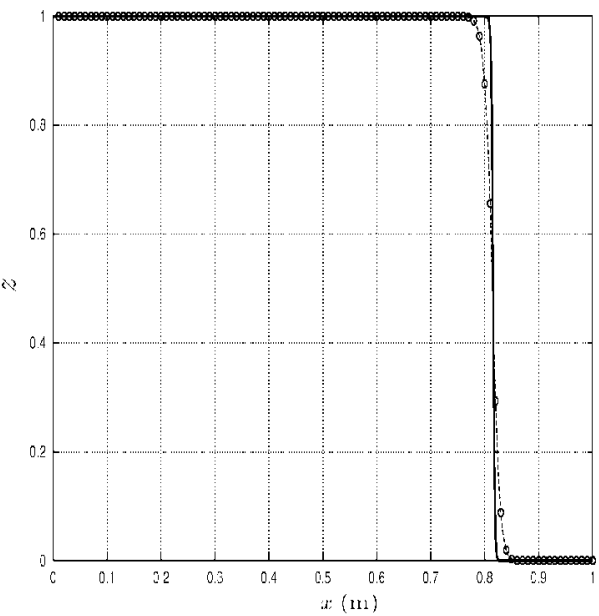

Pressure

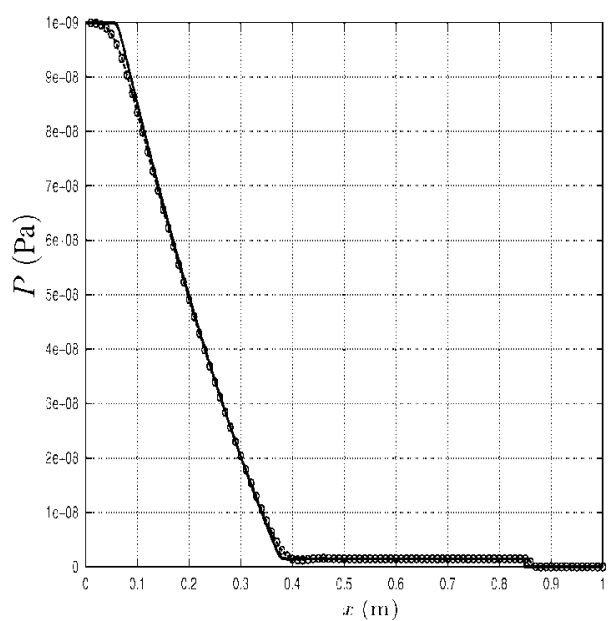

Density

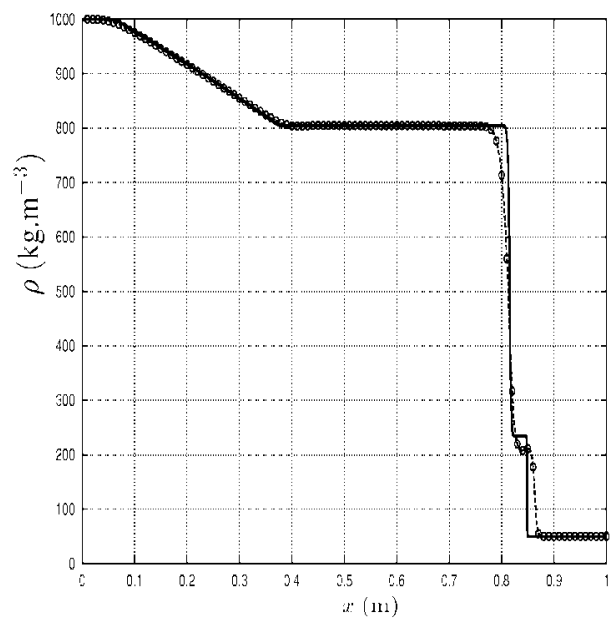

Velocity

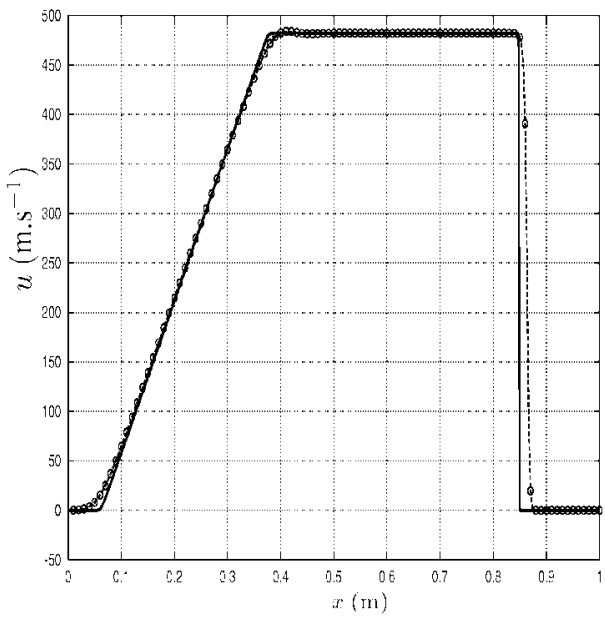

FIG. 4. Shock tube test problem between a stiffened gas and a van der Waals gas: results for a fine grid (solid line) and a coarse grid (dotted line).

ones, and only our second scheme (68)-(71), for the advection of the color function $z$, gives good results. Our solver does not handle perfectly the rarefaction on the right side of the tube: there is a slight undershoot of the pressure (with even negative values; see Fig. 5). Recall that, although negative values of the pressure are not physically relevant, it is not a

TABLE III

Initial State for Interface-Shock Interaction Problem

\begin{tabular}{lccc}
\hline \multicolumn{1}{c}{ Location } & $\rho\left(\mathrm{kg} \cdot \mathrm{m}^{-3}\right)$ & $u\left(\mathrm{~m} \cdot \mathrm{s}^{-1}\right)$ & $P(\mathrm{~Pa})$ \\
\hline Left (van der Waals) & 1.2 & 0 & $10^{5}$ \\
Middle (stiffened gas): preshock & $10^{3}$ & 0 & $10^{5}$ \\
Right (stiffened gas): postshock & $1.23 \times 10^{3}$ & -432.69 & $10^{9}$ \\
\hline
\end{tabular}


Color function $z$

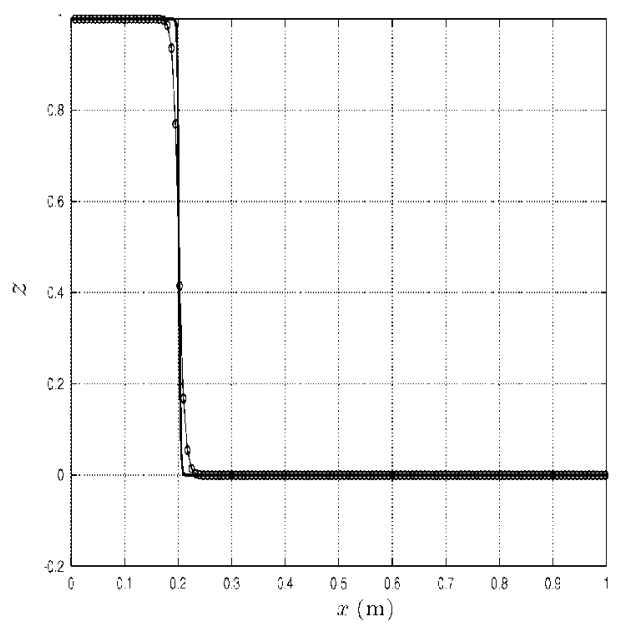

Pressure

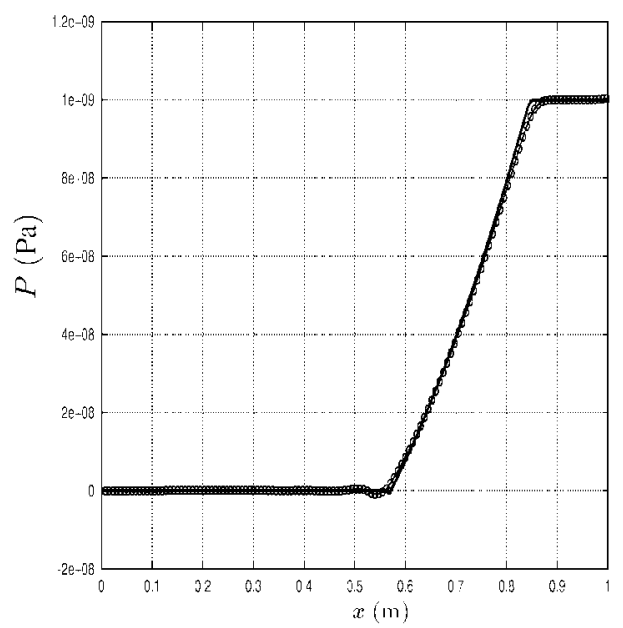

Density

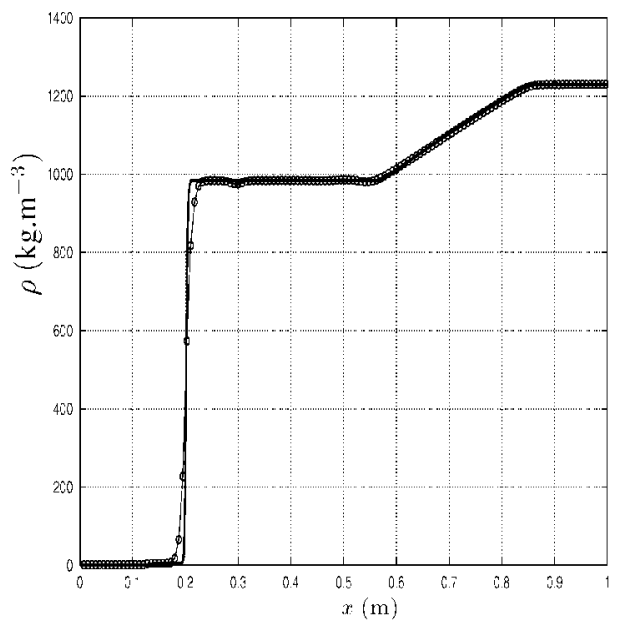

Volocity

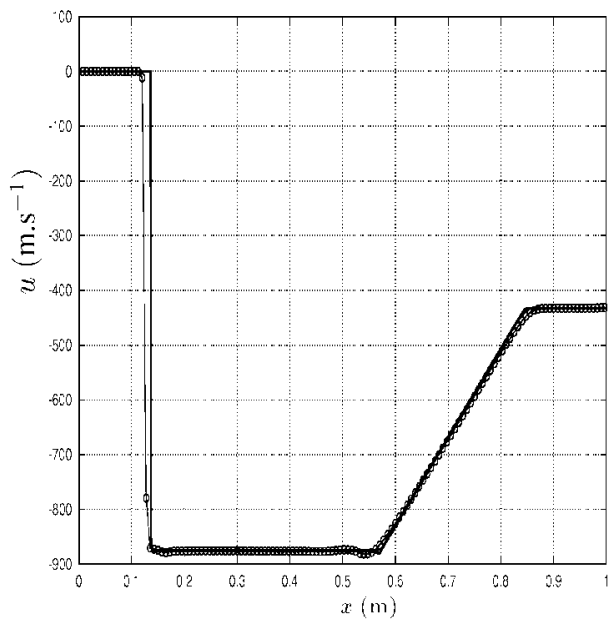

FIG. 5. Computational results for the 1-D shock-interface test with a van der Waals gas and a stiffened gas for a fine grid (solid line) and a coarse grid (dotted line).

problem for a stiffened gas as long as its internal energy and squared sound velocity remain positive (which is the case here). Let us note that these numerical flaws seem to fade away with grid refinement.

\subsection{Impact Problem}

The fifth test (originating from [25]) is an impact problem between two materials (copper and an inert explosive) following the Cochran-Chan EOS we introduced in Section 8.1 (a special type of Mie-Gruneisen EOS already described in Section 8.1). The copper (on the left) impacts the inert explosive (at rest on the right) with a velocity of $1500 \mathrm{~m} \cdot \mathrm{s}^{-1}$ under atmospheric pressure. The details of the numerical parameters of this test are given in Table II. The exact solution is made of two shocks propagating in opposite directions. 


\section{Color function z}

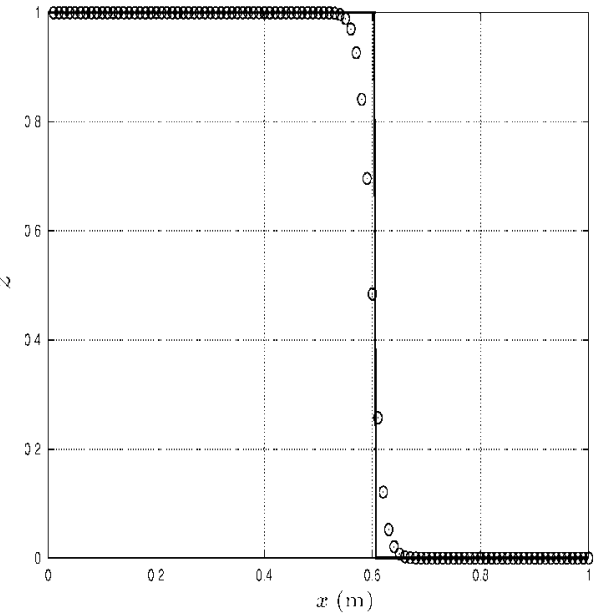

Pressure

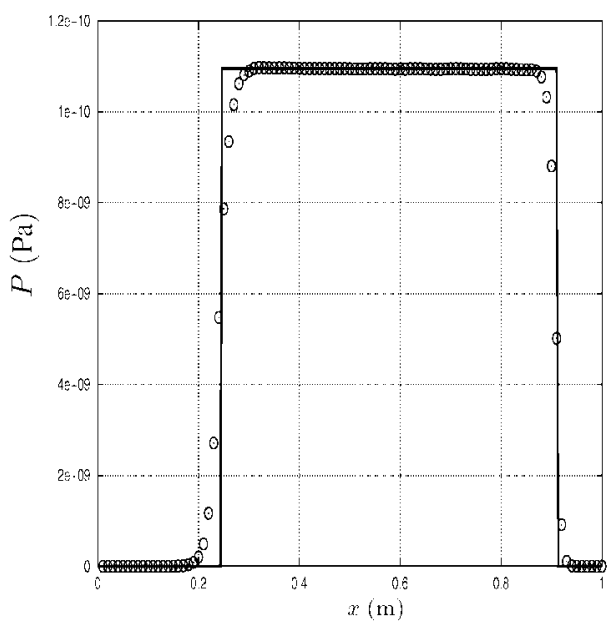

Density

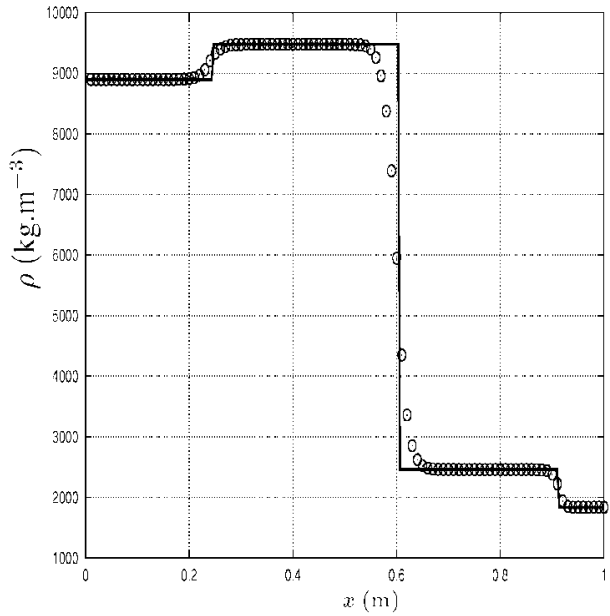

Velocity

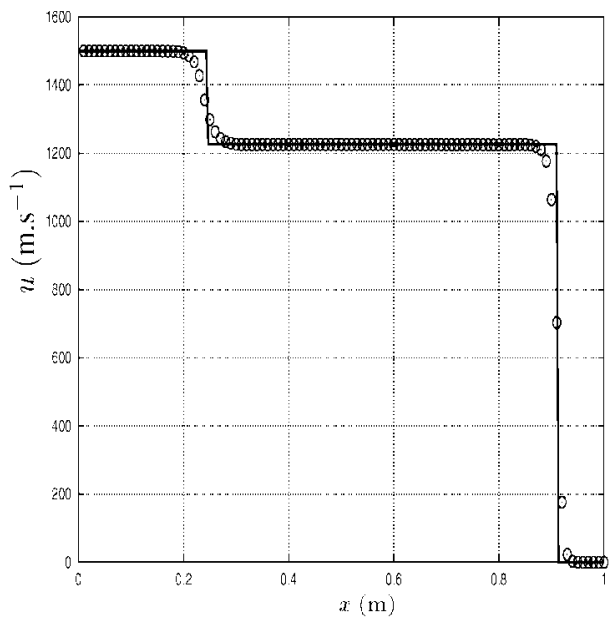

FIG. 6. Exact (solid line) and numerical solutions (dotted line) of an impact problem between two MieGruneisen materials.

The domain is $1-\mathrm{m}$ long and is discretized with 100 cells. The interface between the materials is initially located at $x=0.5 \mathrm{~m}$. The flow variables are displayed at $t=85 \mu \mathrm{s}$ in Fig. 6. Our results are very similar to those obtained in [25].

\subsection{Tabulated EOS Tests}

An interesting feature of the five-equation model is that no analytical form of the fluid EOS is required. We therefore perform two numerical tests to demonstrate this possibility. The use of tabulated EOSs is very common in industry. However, the data of such tabulated EOSs is not readily available (it is already a huge task to enter all the numerical values in a file). For simplicity and for ease of reproducibility, we tabulated the van der Waals gas EOS of Section 8.3. 
The tabulation process has been performed with a $1000 \times 1000$ uniform discretization of the $(\rho, P)$ plane limited by $0 \leq \rho \leq 990$ and $10^{4} \leq P \leq 10^{9}$. Values of $\rho \varepsilon$ are given at each $(\rho, P)$ node. The function $(\rho, P) \mapsto \rho \varepsilon(\rho, P)$ is provided by a $Q_{1}$ interpolation of the $\rho \varepsilon$ table values. Thus, the function $(\rho, \varepsilon) \mapsto P(\rho, \varepsilon)$ is obtained implicitly owing to a Newton method. The isobaric closure $P_{1}=P_{2}$ is used as in Proposition 4.1 to recover all the thermodynamic variables fron the conservative variables. More precisely, we solve the equation $\left(P_{1}-P_{2}\right)\left(\rho_{1} \varepsilon_{1}\right)=0$ with a dichotomy algorithm.

We first perform an advection test between this "tabulated EOS" fluid and a stiffened gas (the same as that of Section 8.3). The tabulated EOS fluid is initially located on the left side of a $1-\mathrm{m}$ tube for $0<x<0.5$ with a density $\rho=50 \mathrm{~kg} \cdot \mathrm{m}^{-3}$. The right side $0.5<x<1$ contains the same stiffened gas as in Section 8.3 , with $\rho=1000 \mathrm{~kg} \cdot \mathrm{m}^{-3}$. Pressure and velocity are uniformly set to $u=1000 \mathrm{~m} \cdot \mathrm{s}^{-1}$ and $P=10^{5} \mathrm{~Pa}$. Figure 7

Color function z

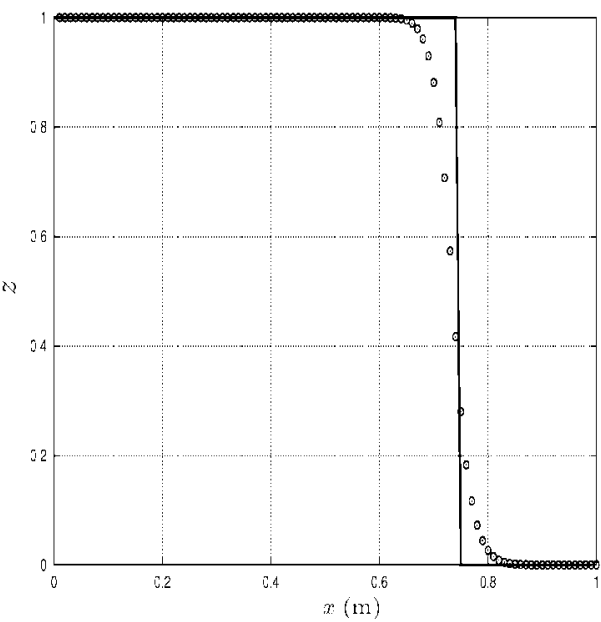

Prossure

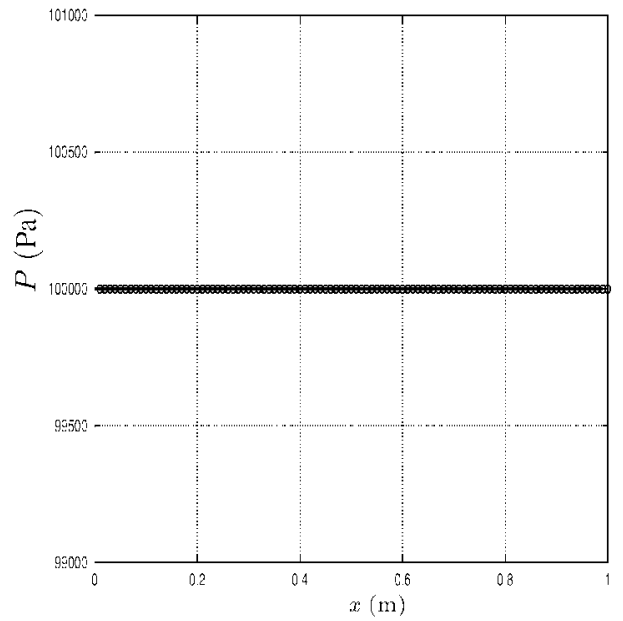

Density

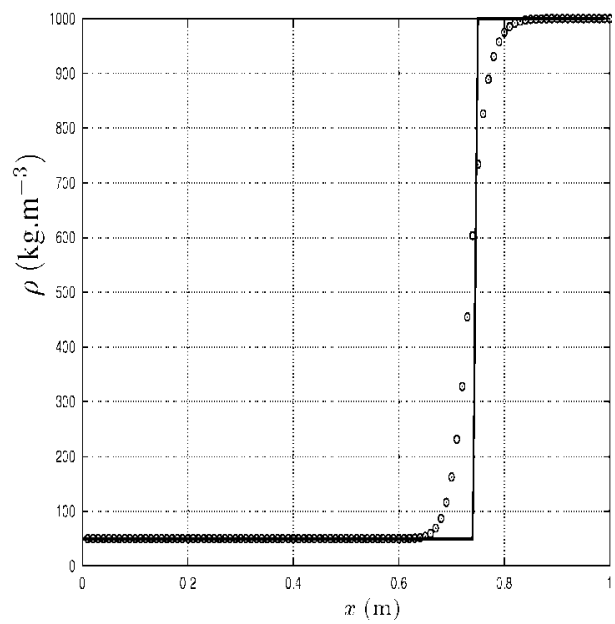

Volocity

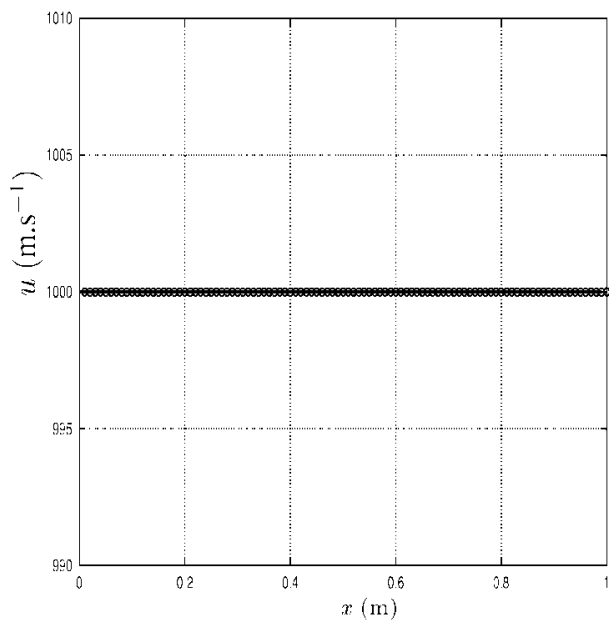

FIG. 7. Interface advection problem between a stiffened gas and a tabulated EOS fluid: exact (solid line) and numerical (dotted line) results. 
Color function $z$

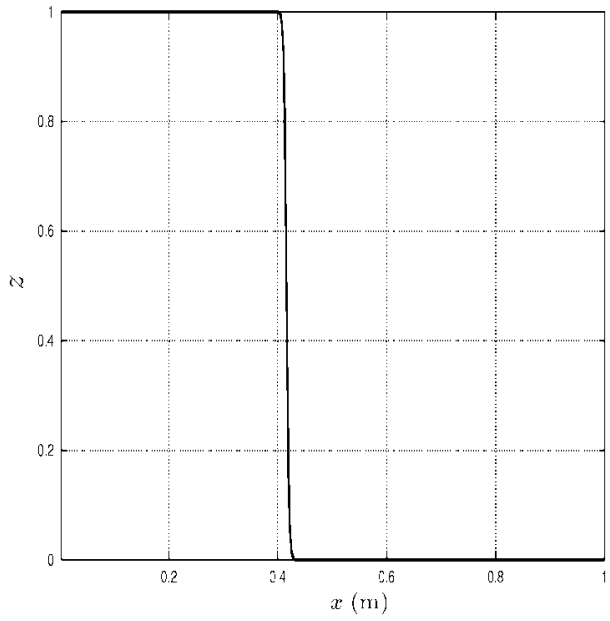

Pressure

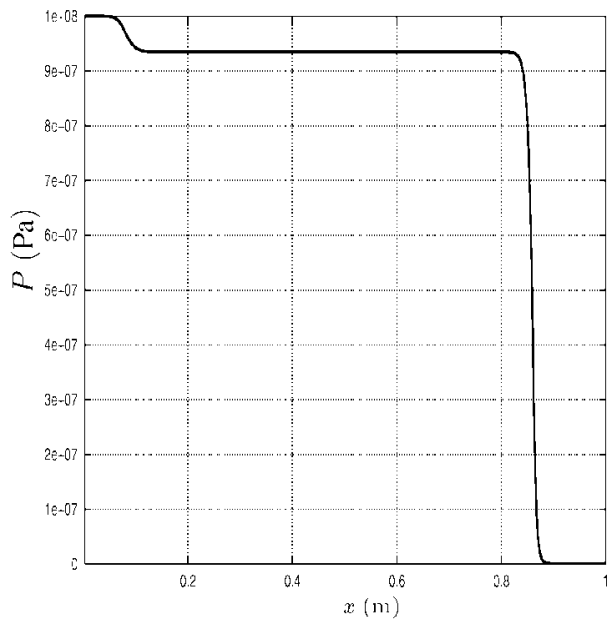

Density

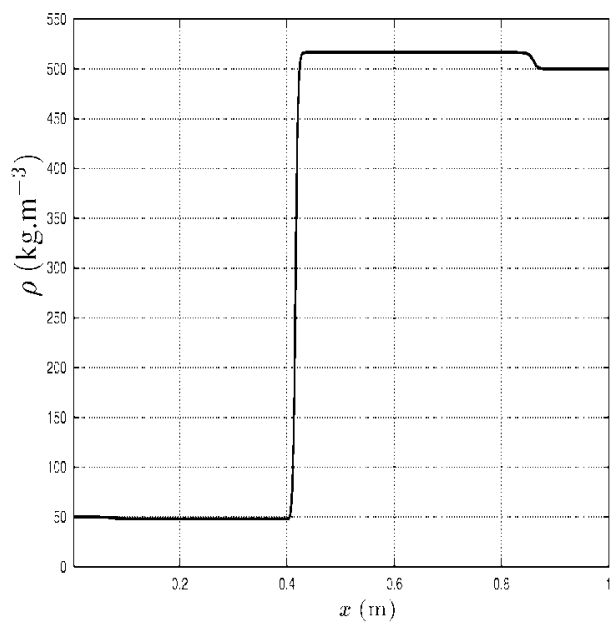

Velocity

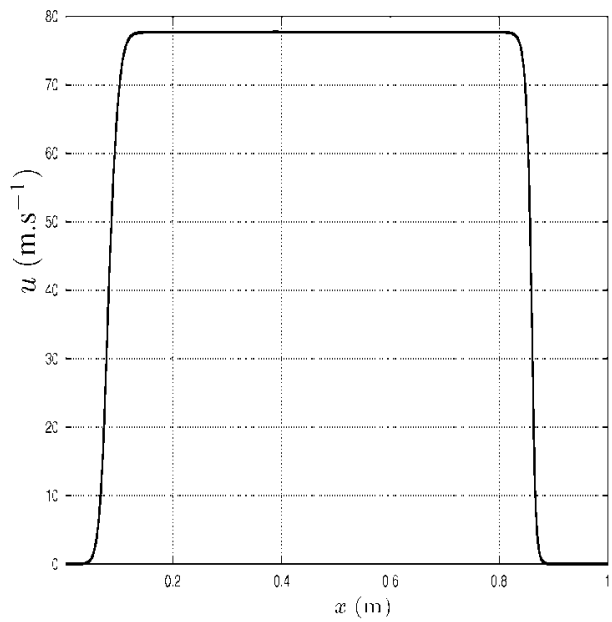

FIG. 8. Shock tube problem between a stiffened gas and a tabulated EOS fluid.

displays the solution computed on a 100-cell grid, with constant states maintained on the left and right boundaries, at $t=240 \mu \mathrm{s}$. The analytical solution is also plotted. We see that both the pressure and velocity constant profiles are, as expected, preserved by the solver.

Second we perform a shock tube test with the same two fluids. They are initially at rest, on each side of an interface lying at $x=0.4 \mathrm{~m}$ in a $1-\mathrm{m}$ tube. On the left side, the tabulated EOS of the fluid is given by $\rho=50 \mathrm{~kg} \cdot \mathrm{m}^{-3}$ and $P=10^{8} \mathrm{~Pa}$. On the right side we have $\rho=500 \mathrm{~kg} \cdot \mathrm{m}^{-3}$ and $P=10^{5} \mathrm{~Pa}$ for the stiffened gas. Figure 8 displays the computed solution at $t=190 \mu$ s performed with a 400-cell grid.

\subsection{Bubble-Shock Interaction}

Our last test is a well-known 2-D problem (see, e.g., [22, 30]) of bubble and shock interaction (the previous shock-interface interaction test is its 1-D version). We extend 


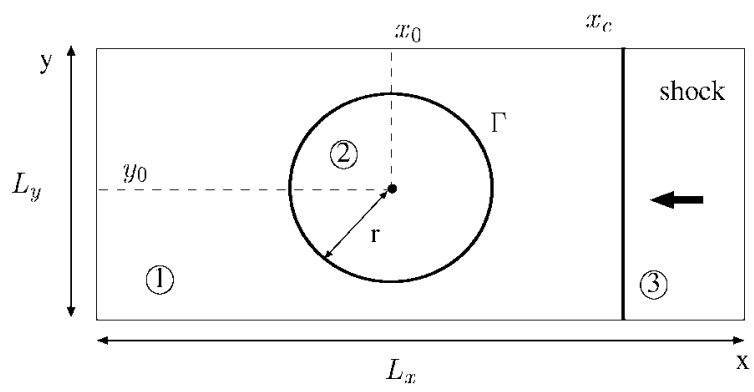

FIG. 9. The shock-bubble interaction case test.

our previous second-order 1-D scheme with a classical dimensional splitting strategy on a regular grid. The geometry of the problem is displayed in Fig. 9. The domain is a rectangle of size $L_{x}=1.2 \mathrm{~m}$ and $L_{y}=1 \mathrm{~m}$. A $480 \times 400$ mesh is used for the discretization. A bubble of a van der Waals fluid with a radius $r=0.2 \mathrm{~m}$ initially located at $\left(x_{0}, y_{0}\right)=(0.7,0.5)$ is surrounded by a stiffened gas. This gas and the bubble are in equilibrium. A planar Mach 1.422 shock originally located at $x_{c}=0.95 \mathrm{~m}$ travels in the stiffened gas from the right side of the domain and hits the bubble. On the upper and lower boundary reflecting conditions are imposed, while a far-field condition is maintained on the left and right boundaries.

The initial data for the problems are gathered in Table IV. For this table and Fig. 9, the state (1) refers to the preshock state in the stiffened gas, (2) refers to the van der Waals fluid bubble, and (3) denotes the postshock stiffened gas state. As previously the EOS for both fluids are given by relations (72) and (73).

The shock propagates first through the stiffened gas before it hits the bubble, which gains speed and loses its circular shape. This appears readily in Fig. 10, which displays the color function as well as the pressure and density fields. Figure 11 shows that a small pair of vortices appears on the tail of the bubble, increasing the pattern complexity of the interface.

Figure 10 also shows that the shock loses its 1-D character when it hits the bubble, creating circular pressure waves. These waves are reflected by the domain boundaries and hit the vortex again.

Figure 12 displays the Schlieren diagram of the test which corresponds to the graph of $|\operatorname{grad} \rho|$. This graph is especially of interest as it reflects what can be observed during a real physical experiment when a light beam is projected through the fluid (see [22]). We added to the graph the color function isovalues to indicate the bubble position.

Finally, we provide the cross-section graphs (Fig. 13) along the line $x=0.5 \mathrm{~m}$ for the pressure and the density, which show a very good quantitative agreement with the solution computed by Shyue in [30]. Let us note that a wave moving to the right side of the domain can be observed on the density isovalues profile (Fig. 10) and the Schlieren diagram (Fig. 12),

TABLE IV

Initial State for the Shock-Bubble Interaction Test

\begin{tabular}{lccc}
\hline \multicolumn{1}{c}{ Location } & $\rho\left(\mathrm{kg} \cdot \mathrm{m}^{-3}\right)$ & $u\left(\mathrm{~m} \cdot \mathrm{s}^{-1}\right)$ & $P(\mathrm{~Pa})$ \\
\hline (1) Stiffened gas (preshock) & $10^{3}$ & 0 & $10^{5}$ \\
(2) Bubble & 1.2 & 0 & $10^{5}$ \\
(3) Stiffened gas (postshock) & $1.23 \times 10^{3}$ & -432.69 & $10^{9}$ \\
\hline
\end{tabular}


Density
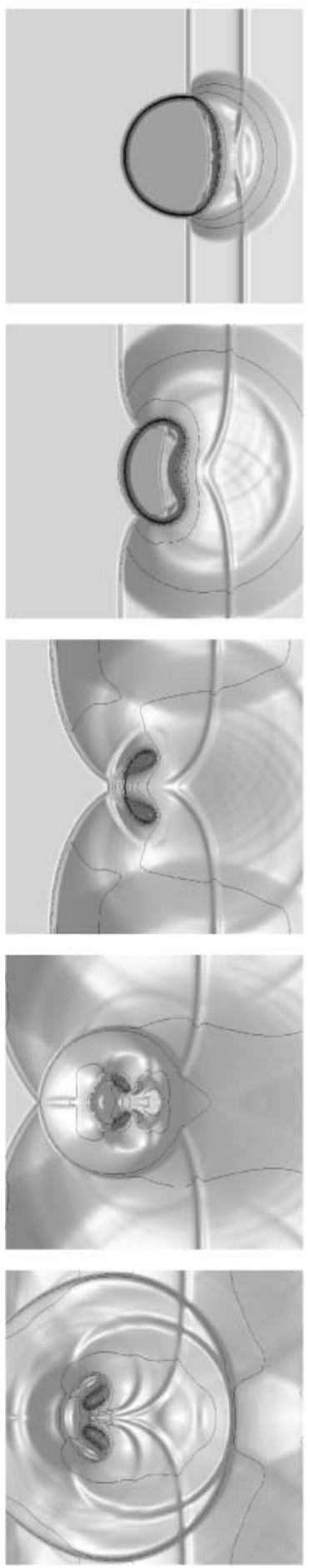

Pressure
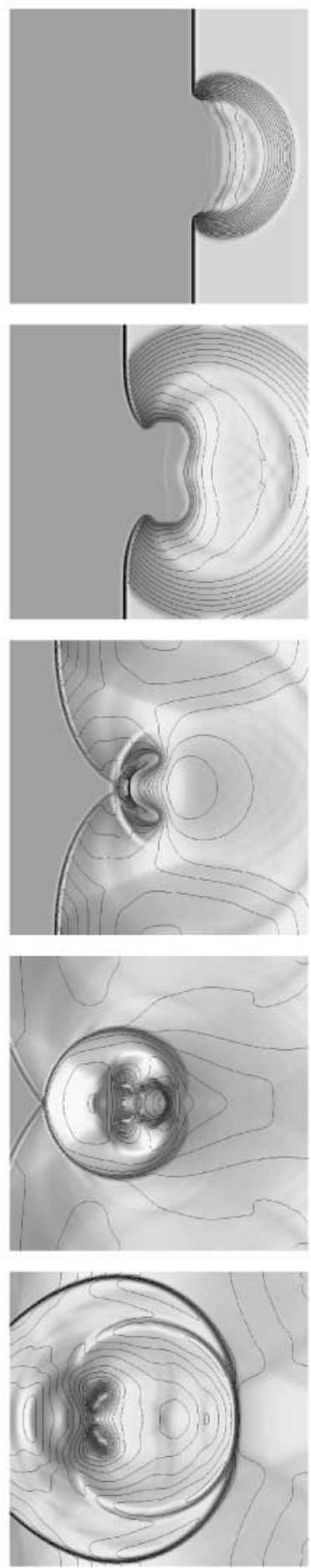

Color Function
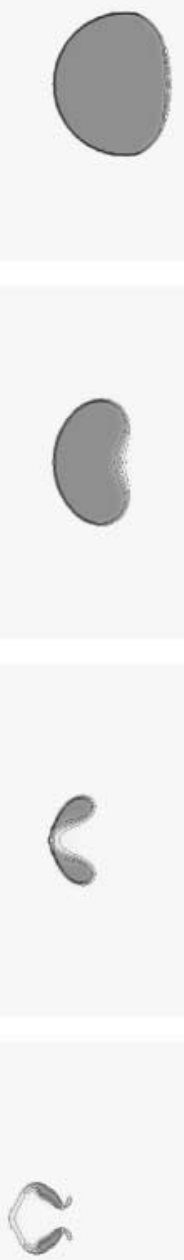

8

FIG. 10. Bubble-shock interaction. Results at instants $t=(1,2,3,4,5) \times 10^{-4} \mathrm{~s}$. 
FIG. 11. Bubble-shock interaction. Velocity field profile together with the color function profile at instants $t=(2.5,4.5) \times 10^{-4} \mathrm{~s}$.
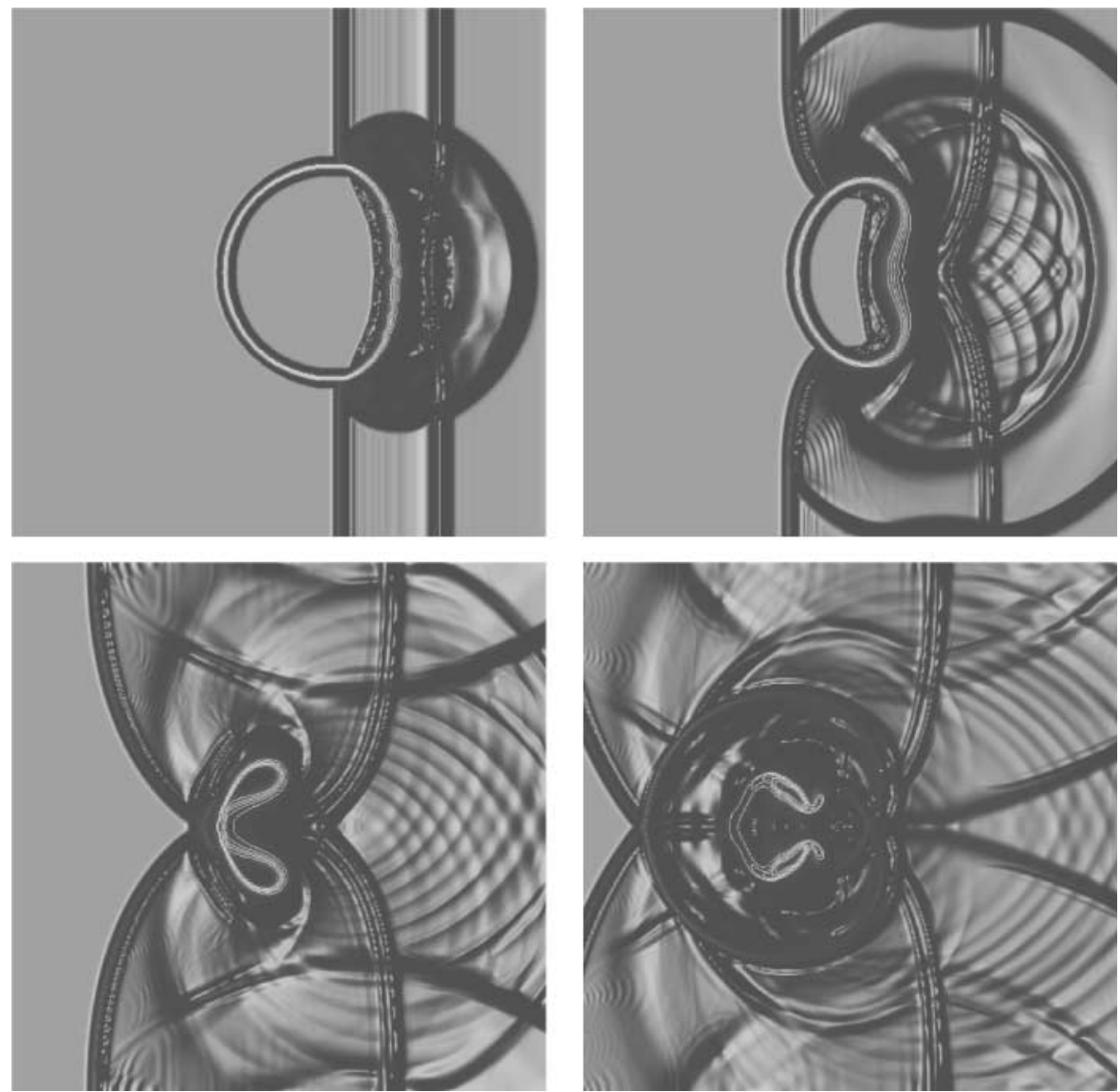

FIG. 12. Bubble-shock interaction. Schlieren diagram at instants $t=(1.2,3.4) \times 10^{-4} \mathrm{~s}$. 

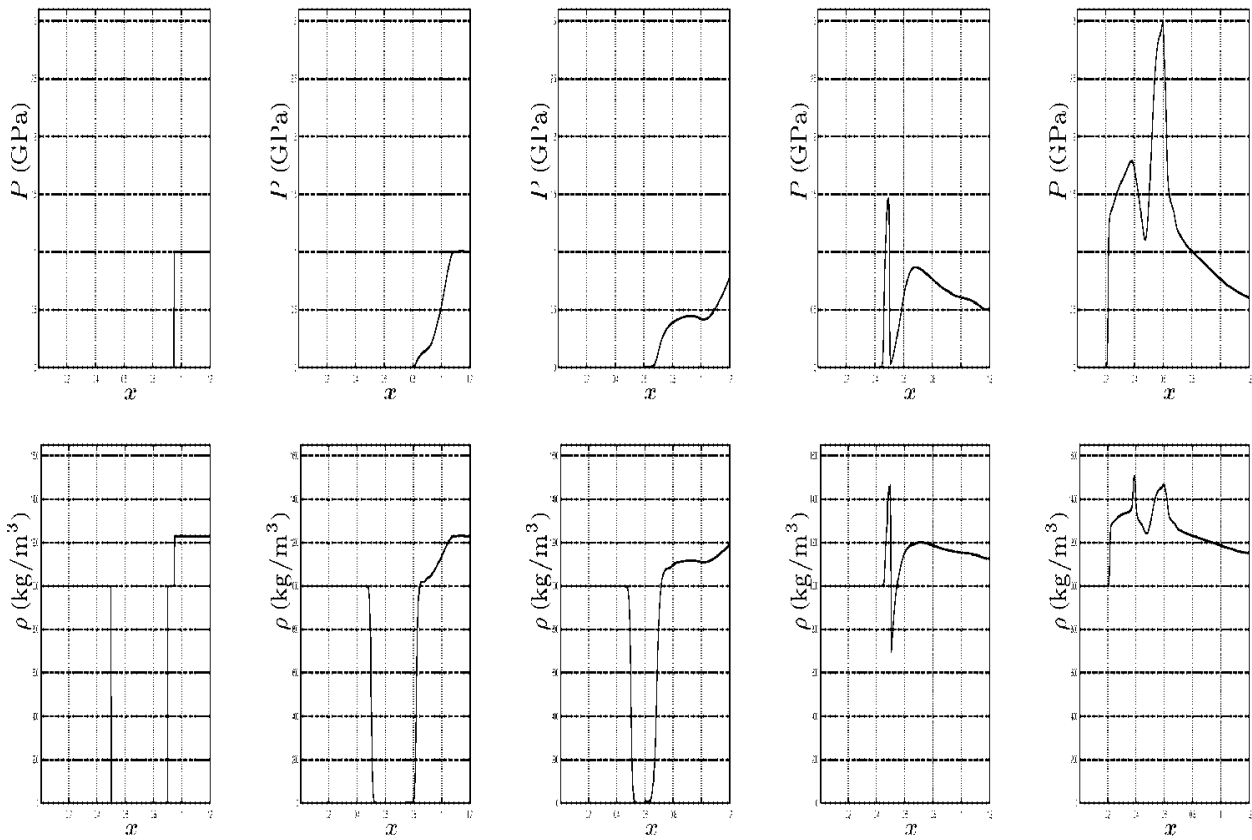

$$
t=0
$$$$
t=100 \mu \mathrm{s}
$$

$t=200 \mu \mathrm{s}$

$$
t=300 \mu \mathrm{s}
$$$$
t=400 \mu \mathrm{s}
$$

FIG. 13. Bubble-shock interaction. Pressure and density along the line $x=0.5 \mathrm{~m}$.

although only a very small glitch can be noticed on the density graph 13 . This wave is the result of a very small inaccuracy in the initial values. It has nevertheless been magnified by the postprocess used for producing the isovalue pictures.

\section{CONCLUSION}

We have presented a five-equation model for the simulation of interfaces between compressible fluids with arbitrary equations of state. This model extends the results of Abgrall [1], Saurel-Abgrall [26], and Shyue [29] for perfect, stiffened, and van der Waals gases. With an isobaric closure, interfaces between fluids can be computed without introducing spurious pressure oscillations. We have proposed a Roe-type numerical method for the simulation of this model. Numerical results demonstrate the efficiency and accuracy of the method. In the future, we plan to improve the robustness of the method in the case of large density ratios and to treat mass transfer phenomena.

\section{REFERENCES}

1. R. Abgrall, How to prevent pressure oscillations in multicomponent flow calculations: A quasi-conservative approach, J. Comput. Phys. 125, 150 (1996).

2. R. Abgrall, Généralisation du schéma de Roe pour le calcul d'écoulements de mélanges de gaz à concentrations variables, Rech. Aerospatiale 6, 31 (1998).

3. R. Abgrall and S. Karni, Computations of compressible multifluids, J. Comput. Phys. 169, 594 (2001).

4. G. Allaire, S. Kokh, and S. Clerc, A 5 equations model for the numerical simulation of interfaces in two-phase flows, C. R. Acad. Sci. Paris, Sér. I 331, 1017 (2000). 
5. S. Clerc, Accurate computation of contact discontinuities in flows with general equations of state, Comput. Meth. Appl. Math. Eng. 178, 245 (1999).

6. S. Clerc, Numerical simulation of the homogeneous equilibrium model for two-phase flows, J. Comput. Phys. 161, 354 (2000).

7. B. després and F. Lagoutière, Un schéma non-linéaire anti-dissipatif pour l'équation d'advection linéaire, C. R. Acad. Sci. Paris, Sér. I 328, 939 (1999).

8. J. Glimm, J. Grove, X. Li, and D. Tan, Robust computational algorithms for dynamic interface tracking in three dimensions, SIAM J. Sci. Comput. 21(6), 2240 (2000).

9. A. Harten and J. M. Hyman, Self adjusting grid methods for one-dimensional hyperbolic conservation laws, J. Comput. Phys. 50, 235 (1983).

10. C. W. Hirt and B. D. Nichols, Volume of fluid (VOF) method for the dynamics of free boundaries, J. Comput. Phys. 39, 201 (1981).

11. S. Karni, Multicomponent flow calculations by a consistent primitive algorithm, J. Comput. Phys. 112, 31 (1994).

12. S. Kokh, Aspects numériques et théoriques de la modélisation des écoulements diphasiques compressibles par des méthodes de capture d'interfaces, Ph.D. thesis (Université Paris 6, 2001).

13. S. Kokh and G. Allaire, Numerical simulation of 2d two-phase flows with interface, in Godunov Methods: Theory and Applications, edited by E. F. Toro (Kluwer Academic/Plenum, Dordrecht/New York, 2001), pp. 513-518.

14. S. Kokh, G. Allaire, and S. Clerc, Towards boiling crisis simulation: The level set method, in Proceedings of the Nureth-9, San Francisco, 1999.

15. B. Lafaurie, C. Nardone, R. Scardovelli, S. Zaleski, and G. Zanetti, Modelling merging and fragmentation in multiphase flows with SURFER, J. Comput. Phys. 113, 134 (1994).

16. F. Lagoutière, Modélisation mathématique et résolution numérique de problèmes de fluides compressibles à plusieurs constituants, Ph.D. thesis (Université Paris VI, 2000).

17. B. Larrouturou, How to preserve the mass fraction positivity when computing compressible multi-component flows, J. Comput. Phys. 95, 59 (1990).

18. X.-D. Liu, R. Fedkiw, and S. Osher, A quasi-conservative approach to the multiphase euler equations without spurious pressure oscillations, in Advances in Scientific Computing, edited by Z.-C. Shi, M. Mu, W. Xue, and J. Zou (Science Press, Beijing/New York, 2001), pp. 106-115.

19. J. Massoni, R. Saurel, B. Nkonga, and R. Abgrall, Proposition de méthodes et modèles Eulériens pour les problèmes à interfaces entre fluides compressibles en présence de transfert de chaleur, Int. J. Heat Mass Transfer 45(6), 1287 (2001).

20. S. Osher and J. Sethian, Front propagating with curvature dependent speed: Algorithms based on HamiltonJacobi formulations, J. Comput. Phys. 78, 12 (1988).

21. S. Osher and P. Smereka, A level set approach for computing solutions to incompressible two-phase flow, J. Comput. Phys. 114, 146 (1994).

22. J. J. Quirk and S. Karni, On the dynamics of a shock-bubble interaction, J. Fluid Mech. 318, 129 (1996).

23. P. L. Roe, Approximate Riemann solvers, parameter vectors, and difference schemes, J. Comput. Phys. 43, 357 (1981).

24. L. Sainsaulieu, Finite volume approximation of two phase-fluid flows based on an appromixate Roe-type Riemann solver, J. Comput. Phys. 121, 1 (1995).

25. R. Saurel and R. Abgrall, A multiphase Godunov method for compressible multifluid and multiphase flows, J. Comput. Phys. 150, 425 (1999).

26. R. Saurel and R. Abgrall, A simple method for compressible multifluid flows, SIAM J. Sci. Comput. 21(3), 1115 (1999).

27. R. Scardovelli and S. Zaleski, Direct numerical simulation of free-surface and interfacial flow, Annu. Rev. Fluid Mech. 31, 567 (1999).

28. J. A. Sethian, Level Set Methods and Fast Marching Methods: Evolving Interfaces in Computational Geometry, Fluid Mechanics, Computer Vision and Materials Science (Cambridge Univ. Press, Cambridge, UK, 1999). 
29. K. M. Shyue, An efficient shock-capturing algorithm for compressible multicomponent problems, J. Comput. Phys. 142, 208 (1998).

30. K. M. Shyue, A fluid-mixture type algorithm for compressible multicomponent flow with van der Waals equation of state, J. Comput. Phys. 156, 43 (1999).

31. K. M. Shyue, A fluid-mixture type algorithm for compressible multicomponent flow with Mie-Gruneisen equation of state, J. Comput. Phys. 171, 678 (2001).

32. H. Stewart and B. Wendroff, Two-phase flow: Models and methods, J. Comput. Phys. 56, 363 (1984).

33. S. O. Unverdi and G. Tryggvason, A front tracking method for viscous, incompressible, multifluid flows, J. Comput. Phys. 100, 25 (1992). 